\title{
BLOCK FETI-DP/BDDC PRECONDITIONERS FOR MIXED ISOGEOMETRIC DISCRETIZATIONS OF THREE-DIMENSIONAL ALMOST INCOMPRESSIBLE ELASTICITY
}

\author{
O. B. WIDLUND *, L. F. PAVARINO ${ }^{\dagger}$, S. SCACCHI ${ }^{\ddagger}$, AND S. ZAMPINI $\S$
}

March 25, 2019

\begin{abstract}
A block FETI-DP/BDDC preconditioner for mixed formulations of almost incompressible elasticity is constructed and analyzed; FETI-DP (dual primal finite element tearing and interconnection) and BDDC (balancing domain decomposition by constraints) are two very successful domain decomposition algorithms for a variety of elliptic problems. The saddle point problems of the mixed problems are discretized with mixed isogeometric analysis with continuous pressure fields. As in previous work by $\mathrm{Tu}$ and $\mathrm{Li}$ (2015), for finite element discretizations of the incompressible Stokes system, the proposed preconditioner is applied to a reduced positive definite system involving only the pressure interface variable and the Lagrange multipliers of the FETI-DP algorithm. The novelty of this preconditioner consists in using BDDC with deluxe scaling for the interface pressure block as well as deluxe scaling for the FETI-DP preconditioner for the Lagrange multiplier block. A convergence rate analysis is presented with a condition number bound for the preconditioned operator which depends on the inf-sup parameter of the fully assembled problem and the condition number of a closely related BDDC algorithm for compressible elasticity. This bound is scalable in the number of subdomains, poly-logarithmic in the ratio of subdomain and element sizes, and robust with respect to material incompressibility and presence of discontinuities of the Lamé parameters across subdomain interfaces. Parallel numerical experiments validate the theory and indicate how the rate of convergence varies with respect to the spline polynomial degree and regularity and the deformation of the domain. Of particular interest is the development of variants of the algorithm with a coarse component of small dimension.
\end{abstract}

Key words. domain decomposition, FETI-DP and BDDC deluxe preconditioners, isogeometric analysis, NURBS, almost incompressible linear elasticity, continuous pressure fields

AMS subject classifications. 65F $08,65 \mathrm{~N} 30,65 \mathrm{~N} 35,65 \mathrm{~N} 55$

1. Introduction. The development of efficient domain decomposition preconditioners for mixed methods with continuous pressure fields and their analysis have remained an open problem for a number of years. A few years ago, Tu and $\mathrm{Li}$ [44], building on their previous works [31, 32], proposed and analyzed FETI-DP solvers for incompressible Stokes finite element systems with continuous pressure.

In this paper, we extend the theory developed in [44] in two directions, by considering i) more general saddle point problems with a nonzero pressure block, such as for almost incompressible elasticity, (AIE) and ii) isogeometric (IGA) mixed discretizations, see e.g. [11]. The pressure field in our case will also be continuous and

*Courant Institute of Mathematical Sciences, 251 Mercer Street, New York, NY 10012, USA. E-mail and URL: widlund@cims.nyu.edu, http://cs.nyu.edu/cs/faculty/widlund/index.html. This work has been supported by the National Science Foundation Grant DMS-1522736.

${ }^{\dagger}$ Dipartimento di Matematica, Università degli Studi di Pavia, Via Ferrata 5, 27100 Pavia, Italy. E-mail: luca.pavarino@unipv.it. This work was partially supported by the European Research Council through the FP7 Ideas Consolidator Grant HIGEOM n. 616563, by the Italian Ministry of Education, University and Research (MIUR) through the "Dipartimenti di Eccellenza Program 2018-22 - Dept. of Mathematics, University of Pavia", and by the Istituto Nazionale di Alta Matematica (INdAM GNCS), Italy.

‡Dipartimento di Matematica, Università degli Studi di Milano, Via Saldini 50, 20133 Milano, Italy. E-mail: simone.scacchi@unimi.it. This work was supported by grants of M.I.U.R. (PRIN 201289A4LX_002) and of Istituto Nazionale di Alta Matematica (INDAM-GNCS).

$\S$ Extreme Computing Research Center, King Abdullah University of Science and Technology, Thuwal 23955, Saudi Arabia. E-mail: stefano.zampini@kaust.edu.sa. 
in our numerical experiments, we use the isogeometric Taylor-Hood mixed methods as developed in [10].

The outer structure of our dual-primal solver is a block FETI-DP/BDDC preconditioner for a reduced positive definite system involving only the pressure interface variable and a Lagrange multiplier. Instead, the inner structure of our solver differs from that of [44], since the first block of our preconditioner is based on BDDC with deluxe scaling for the interface pressure scaled by the first Lamé parameter, while the second block is based on FETI-DP with deluxe scaling of the Lagrange multiplier.

Our choice of working with deluxe variants of both the FETI-DP and BDDC blocks in our preconditioner is based on our experience with scalar elliptic problems and compressible elasticity where deluxe variants have given us superior performance compare to other scalings such as $\rho$ - and stiffness-scaling; see $[6,7,37]$. For previous work on deluxe scaling for finite elements, we refer to [15, 35, 38, 45, 48, 49, 50]. We note that while the preconditioner for the interface pressure variables can be quite simple for lower order finite elements as those considered by $\mathrm{Tu}$ and $\mathrm{Li}$, the mass matrices for isogeometric analysis can be very ill-conditioned and that a non-trivial preconditioner is required for good performance.

Several arguments in the $\mathrm{Tu}-\mathrm{Li}$ theory also need to be modified when we turn to the AIE case because of the presence of the pressure mass matrices in the mixed formulation. In addition to these modified arguments, our analysis will present a more direct proof of the main condition number bound for the preconditioned operator.

Our choice of primal constraints for the FETI-DP preconditioner is based on our recent results on dual-primal solvers for three-dimensional compressible elasticity, see [37, Theorems 4.3 and 4.5]. We note that these two results are established under different assumptions on the variation of the Lamé parameters across the interface between the subdomains. For simplicity, we will consider only fixed choices of primal constraints, but an adaptive selection of the primal constraints for the FETI-DP method could be considered as well, see, e.g., the general framework in [38] and some specific choices for isogeometric discretizations of scalar elliptic problems in [7]. We note that it is well known that the FETI-DP and BDDC algorithms for elliptic problems are closely related, see, e.g., [33], and that therefore the condition number bounds for the BDDC algorithms obtained in [37] can be used for our FETI-DP algorithm when the primal spaces are the same.

Previous work on domain decomposition methods for Stokes discretizations with continuous pressure can be found in $[8,26,27,41]$, but without a convergence rate analysis. There has also been earlier work by Pavarino and Scacchi [36] on isogeometric discretizations of Stokes problems, also based on [44], but some theoretical issues remained open and the pressure block of the preconditioner was based on the principal minor of the assembled pressure mass matrix related to the values on the interface.

Research on domain decomposition solvers for IGA is a relatively recent field compared with the much older field of finite and spectral element solvers. In addition to our previous works on isogeometric Schwarz and BDDC preconditioners, see [3, 4, 5, $6,7,37$, we mention $[16,18,24]$ on IGA multigrid, [21, 22, 23] on IGA Discontinuous Galerkin methods, and [30, 39] on other IGA solvers. A recent comparison between spectral elements and IGA discretizations and solvers can be found in [19].

The rest of the paper is organized as follows. The problem of almost incompressible elasticity is introduced in Section 2 and its isogeometric discretization in Section 3. A dual-primal decomposition leading to a reduced system is described in Section 4, while the block FETI-DP/BDDC preconditioner is described in Section 5. Our 
convergence rate analysis is presented in Section 6 and the paper is concluded by Section 7 with results from some of our numerical experiments.

2. The almost incompressible elasticity systems. Let $\Omega$ be a domain in $\mathbb{R}^{3}$ which can be represented exactly by the isogeometric analysis system. It is decomposed into $N$ non-overlapping subdomains $\Omega_{i}$, of diameter $H_{i}$, which are images under the geometric map $\boldsymbol{F}$, see Eq. (3.5), of a coarse element partition $\tau_{H}$ of the reference domain. i.e.,

$$
\bar{\Omega}=\bigcup_{i=1}^{N} \bar{\Omega}_{i}
$$

The interface of this decomposition (2.1) is given by $\Gamma=\left(\bigcup_{i=1}^{N} \partial \Omega_{i}\right) \backslash \partial \Omega$. In the next subsection, we will further partition each subdomain into many shape-regular elements. We will assume that the nodes match across the interface between the subdomains.

We will use the standard notation $L^{2}(\Omega)$ to denote the space of square integrable functions on any open set $\Omega$, and by $H^{1}(\Omega)$ the classical Sobolev space of order 1 of functions that are in $L^{2}(\Omega)$ with first derivatives in $L^{2}(\Omega)$.

The boundary $\partial \Omega$ is the union of two disjoint sets $\partial \Omega_{D}$ and $\partial \Omega_{N}$ where $\partial \Omega_{D}$ is of non-zero surface measure. We will work with two load functions $\mathbf{g} \in\left[L^{2}(\Omega)\right]^{3}$ and $\mathbf{g}_{N} \in\left[L^{2}\left(\partial \Omega_{N}\right)\right]^{3}$, and the spaces

$$
\mathbf{V}:=\left\{\boldsymbol{v} \in H^{1}(\Omega)^{3}:\left.\boldsymbol{v}\right|_{\partial \Omega_{D}}=0\right\}, \quad Q:=L^{2}(\Omega)
$$

The load functions define a linear functional $<\mathbf{f}, \boldsymbol{v}>:=\int_{\Omega} \mathbf{g} \cdot \boldsymbol{v} d x+\int_{\partial \Omega_{N}} \mathbf{g}_{N} \cdot \boldsymbol{v} d A$.

We will work with a mixed formulation of linear elasticity for almost incompressible materials as, e.g., in [9, Ch. 1]: find the material displacement $\mathbf{u} \in \mathbf{V}$ and the pressure $p \in Q$ such that

$$
\left\{\begin{array}{cccc}
2 \int_{\Omega} \mu \varepsilon(\mathbf{u}): \varepsilon(\boldsymbol{v}) d x & -\int_{\Omega} \operatorname{div} \boldsymbol{v} p d x & =<\mathbf{f}, \boldsymbol{v}> & \forall \boldsymbol{v} \in \mathbf{V} \\
-\int_{\Omega} \operatorname{div} \mathbf{u} q d x-\int_{\Omega} \frac{1}{\lambda} p q d x= & 0 & \forall q \in Q
\end{array}\right.
$$

Here $\varepsilon$ is the symmetric gradient operator and $\mu(x)$ and $\lambda(x)$ the Lamé parameters of the material that for simplicity, when developing the theory, are assumed to be constant in each subdomain $\Omega_{i}$, i.e. $\mu=\mu_{i}$ and $\lambda=\lambda_{i}$ in $\Omega_{i}$. These parameters can be expressed in terms of the local Poisson ratio $\nu_{i}$ and Young's modulus $E_{i}$ as

$$
\mu_{i}:=\frac{E_{i}}{2\left(1+\nu_{i}\right)}, \quad \lambda_{i}:=\frac{E_{i} \nu_{i}}{\left(1+\nu_{i}\right)\left(1-2 \nu_{i}\right)}
$$

The material of a subdomain approaches the incompressible limit when $\nu_{i} \rightarrow 1 / 2$.

Factoring out the constants $\mu_{i}$ and $\frac{1}{\lambda_{i}}$, we can define local bilinear forms in terms 
of integrals over the subdomains $\Omega_{i}$ and obtain

$$
\begin{aligned}
\mu a(\mathbf{u}, \boldsymbol{v}) & :=\sum_{i=1}^{N} \mu_{i} a_{i}(\mathbf{u}, \boldsymbol{v}):=\sum_{i=1}^{N} 2 \mu_{i} \int_{\Omega_{i}} \varepsilon(\mathbf{u}): \varepsilon(\boldsymbol{v}) d x \\
b(\boldsymbol{v}, q) & :=\sum_{i=1}^{N} b_{i}(\boldsymbol{v}, q):=-\sum_{i=1}^{N} \int_{\Omega_{i}} \operatorname{div} \boldsymbol{v} q d x, \\
\frac{1}{\lambda} c(p, q) & :=\sum_{i=1}^{N} \frac{1}{\lambda_{i}} c_{i}(p, q):=\sum_{i=1}^{N} \frac{1}{\lambda_{i}} \int_{\Omega_{i}} p q d x .
\end{aligned}
$$

We note that in the general case of piecewise constants parameters, $\mu a(\boldsymbol{u}, \boldsymbol{v})$ and $\frac{1}{\lambda} c(p, q)$ are only symbolic notations representing the bilinear forms defined above, obtained by sub-assembling of bilinear forms originating from the subdomains. The mixed elasticity problem (2.2) can then be written: find $\mathbf{u} \in \mathbf{V}, p \in Q$ such that

$$
\left\{\begin{array}{ccccc}
\mu a(\mathbf{u}, \boldsymbol{v}) & +b(\boldsymbol{v}, p) & = & <\mathbf{f}, \boldsymbol{v}> & \forall \boldsymbol{v} \in \mathbf{V} \\
b(\mathbf{u}, q)- & \frac{1}{\lambda} c(p, q) & = & 0 & \forall q \in Q .
\end{array}\right.
$$

3. Isogeometric discretization of the mixed elasticity problem. We are now ready to present the isogeometric approximation of the mixed elasticity problem (2.2). In the present description, we consider, for simplicity of exposition, only the homogeneous Dirichlet case $\partial \Omega_{D}=\partial \Omega$ that can be obtained, as observed for instance in [2], by eliminating the first and last functions in each coordinate. To simplify the presentation, we will also only consider the case when the reference domain is a unit cube rather than the union of more than one cuboid.

We will use univariate B-spline basis functions $L_{i}^{p}(\xi)$ of degree $p$ associated to the knot vector $\left\{\xi_{1}=0, \ldots, \xi_{\ell+p+1}=1\right\}$ defined on the parametric interval $\widehat{I}:=(0,1)$. Given analogous univariate functions $M_{j}^{q}(\eta)$ and $N_{k}^{r}(\zeta)$, we define by a tensor product the three-dimensional (3D) parametric space on $\widehat{\Omega}:=(0,1) \times(0,1) \times(0,1)$, the $\ell \times m \times n$ mesh of control points $\mathbf{C}_{i, j, k}$ associated with the knot vectors $\left\{\xi_{1}=0, \ldots, \xi_{\ell+p+1}=1\right\}$, $\left\{\eta_{1}=0, \ldots, \eta_{m+q+1}=1\right\}$, and $\left\{\zeta_{1}=0, \ldots, \zeta_{n+r+1}=1\right\}$, the trivariate B-spline basis functions by $B_{i, j, k}^{p, q, r}(\xi, \eta, \zeta)=L_{i}^{p}(\xi) M_{j}^{q}(\eta) N_{k}^{r}(\zeta)$, and the trivariate B-spline discrete space by

$$
\widehat{\mathcal{S}}_{h}:=\operatorname{span}\left\{B_{i, j, k}^{p, q, r}(\xi, \eta, \zeta), 1 \leq i \leq \ell, \quad 1 \leq j \leq, m, \quad 1 \leq k \leq n\right\} .
$$

The regularity of these basis functions is determined by the multiplicity of the knots. We note that these basis functions fail to be nodal except for the case of minimal regularity; this will lead to fat interfaces, cf. [4].

Analogously, the NURBS space is the span of NURBS basis functions defined in one dimension by

$$
R_{i}^{p}(\xi):=\frac{L_{i}^{p}(\xi) \omega_{i}}{\sum_{\hat{i}=1}^{\ell} L_{\hat{i}}^{p}(\xi) \omega_{\hat{i}}}=\frac{L_{i}^{p}(\xi) \omega_{i}}{w(\xi)}
$$

with the weight function $w(\xi):=\sum_{\hat{i}=1}^{\ell} L_{\hat{i}}^{p}(\xi) \omega_{\hat{i}} \in \widehat{\mathcal{S}}_{h}$, and in three dimensions by a tensor product

$$
R_{i, j, k}^{p, q, r}(\xi, \eta, \zeta):=\frac{B_{i, j, k}^{p, q, r}(\xi, \eta, \zeta) \omega_{i, j, k}}{\sum_{\hat{i}=1}^{\ell} \sum_{\hat{j}=1}^{m} \sum_{\hat{k}=1}^{n} B_{\hat{i}, \hat{j}, \hat{k}}^{p, q, r}(\xi, \eta, \zeta) \omega_{\hat{i}, \hat{j}, \hat{k}}}=\frac{B_{i, j, k}^{p, q, r}(\xi, \eta, \zeta) \omega_{i, j, k}}{w(\xi, \eta, \zeta)}
$$


where $w(\xi, \eta, \zeta)$ is the weight function and $\omega_{\hat{i}, \hat{j}, \hat{k}}$ are positive weights associated with a $\ell \times m \times n$ net of control points. The discrete NURBS space on $\Omega$ is then defined as the span of the push-forward of the NURBS basis function, i.e.

$$
\mathcal{N}_{h}:=\operatorname{span}\left\{R_{i, j, k}^{p, q, r} \circ \mathbf{F}^{-1}, \text { with } 1 \leq i \leq \ell, \quad 1 \leq j \leq m, 1 \leq k \leq n\right\},
$$

with $\mathbf{F}: \widehat{\Omega} \rightarrow \Omega$, the geometrical map between the parameter and physical spaces given by

$$
\mathbf{F}(\xi, \eta, \zeta)=\sum_{i=1}^{\ell} \sum_{j=1}^{m} \sum_{k=1}^{n} R_{i, j, k}^{p, q, r}(\xi, \eta, \zeta) \mathbf{C}_{i, j, k} .
$$

We then introduce the spline space on the parameter space $\widehat{\mathbf{V}}_{h}=\left[\mathcal{S}_{h} \cap H_{0}^{1}(\widehat{\Omega})\right]^{3}$ as

$$
\widehat{\mathbf{V}}_{h}:=\left[\operatorname{span}\left\{B_{i, j, k}^{p, q, r}: 2 \leq i \leq \ell-1,2 \leq j \leq m-1,2 \leq k \leq n-1\right\}\right]^{3}
$$

and the NURBS space defined in physical space $\mathbf{V}_{h}=\left[\mathcal{N}_{h} \cap H_{0}^{1}(\Omega)\right]^{3}$ as

$$
\mathbf{V}_{h}:=\left[\operatorname{span}\left\{R_{i, j, k}^{p, q, r} \circ \mathbf{F}^{-1}: 2 \leq i \leq \ell-1,2 \leq j \leq m-1,2 \leq k \leq n-1\right\}\right]^{3} .
$$

In order to discretize our saddle point problems, we need a proper coupling between the mapped NURBS displacement space $\mathbf{V}_{h}$ and the pressure space $Q_{h}$, as for the finite element case developed in $[9$, Ch. 8]. Here we consider the following pair of spaces based on the isogeometric Taylor-Hood elements introduced in [10], that have proven to be inf-sup stable. The space $\mathbf{V}_{h}$ is built as in (3.6), but with the restrictions that

- the polynomial degrees satisfy $p \geq 2, q \geq 2, r \geq 2$;

- all the knots are repeated at least twice, and therefore the space is at most $C^{p-2}-C^{q-2}-C^{r-2}$ regular across mesh lines.

The space $Q_{h}$ is built as the span

$$
Q_{h}=\operatorname{span}\left\{\mathrm{R}_{i, j, k}^{p-1, q-1, r-1} \circ \mathbf{F}^{-1} \text {, with } 1 \leq i \leq \bar{\ell}, \quad 1 \leq j \leq \bar{m}, \quad 1 \leq k \leq \bar{n}\right\},
$$

where the basis $\mathrm{R}_{i, j, k}^{p-1, q-1, r-1}$ is generated from the same knot vectors as for the $\mathbf{V}_{h}$ space, but with one less repetition for each multiple knot, so that $\bar{\ell}, \bar{m}, \bar{n}$ are approximately one half of $\ell, m, n$, respectively. Therefore, the space $Q_{h}$ of pressure will have the same regularity across mesh lines as $\mathbf{V}_{h}$, but with a polynomial degree one less. In the case of the same polynomial degree $p$ for each coordinate, we will then consider isogeometric Taylor-Hood elements with displacement basis functions of degree $p$, regularity $p-2$ and pressure basis functions of degree $p-1$, regularity $p-2$. Mixed spaces with lower regularity can be considered as well.

The IGA approximation of our model Stokes or mixed elasticity problem (2.7) now reads: find $\mathbf{u}_{h} \in \mathbf{V}_{h}, p_{h} \in Q_{h}$ such that

$$
\left\{\begin{array}{ccccc}
\mu a\left(\mathbf{u}_{h}, \boldsymbol{v}_{h}\right)+b\left(\boldsymbol{v}_{h}, p_{h}\right) & =<\mathbf{f}, \boldsymbol{v}_{h}> & \forall \boldsymbol{v}_{h} \in \mathbf{V}_{h}, \\
b\left(\mathbf{u}_{h}, q_{h}\right)-\frac{1}{\lambda} c\left(p_{h}, q_{h}\right) & = & 0 & \forall q_{h} \in Q_{h}
\end{array}\right.
$$

Denoting with the same symbols $\mathbf{u}_{h}, p_{h}$ both the isogeometric functions and their vector representations in the isogeometric basis, the matrix form of system (3.8) is

$$
\left[\begin{array}{cc}
\mu A & B^{T} \\
B & -\frac{1}{\lambda} C
\end{array}\right]\left[\begin{array}{l}
\mathbf{u}_{h} \\
p_{h}
\end{array}\right]=\left[\begin{array}{l}
\mathbf{f} \\
0
\end{array}\right]
$$


where $\mu A, B$, and $\frac{1}{\lambda} C$ are matrices associated with the bilinear forms $\mu a(\cdot, \cdot), b(\cdot, \cdot)$, and $\frac{1}{\lambda} c(\cdot, \cdot)$, respectively, and are obtained by sub-assembling the local contributions from the subdomains.

\section{Domain decomposition and a FETI-DP/BDDC reduced system.}

4.1. Dual-Primal decomposition. We will follow [44, Sections 3 and 4] and the notation therein closely. The displacement variables $\mathbf{u}_{h}$ are split into interior $\mathbf{u}_{I}$, dual $\mathbf{u}_{\Delta}$, and primal $\mathbf{u}_{\Pi}$ components, the pressure variables $p_{h}$ into interior $p_{I}$ and interface $p_{\Gamma}$ components, and we denote by $\lambda_{\Delta}$ the Lagrange multipliers used to enforce the continuity of the dual displacements.

Reordering the variables as $\mathbf{u}_{I}, p_{I}, \mathbf{u}_{\Delta}, \mathbf{u}_{\Pi}, p_{\Gamma}$, and $\lambda_{\Delta}$ and splitting the matrices $\mu A, B$, and $\frac{1}{\lambda} C$ into appropriate blocks associated with this splitting, we find that the original system (3.9) is equivalent to

$$
\left[\begin{array}{cccccc}
\mu A_{I I} & B_{I I}^{T} & \mu A_{I \Delta} & \mu A_{I \Pi} & B_{\Gamma I}^{T} & 0 \\
B_{I I} & -\frac{1}{\lambda} C_{I I} & B_{I \Delta} & B_{I \Pi} & -\frac{1}{\lambda} C_{\Gamma I}^{T} & 0 \\
\mu A_{\Delta I} & B_{I \Delta}^{T} & \mu A_{\Delta \Delta} & \mu A_{\Delta \Pi} & B_{\Gamma \Delta}^{T} & B_{\Delta}^{T} \\
\mu A_{\Pi I} & B_{I \Pi}^{T} & \mu A_{\Pi \Delta} & \mu A_{\Pi \Pi} & B_{\Gamma \Pi}^{T} & 0 \\
B_{\Gamma I} & -\frac{1}{\lambda} C_{\Gamma I} & B_{\Gamma \Delta} & B_{\Gamma \Pi} & -\frac{1}{\lambda} C_{\Gamma \Gamma} & 0 \\
0 & 0 & B_{\Delta} & 0 & 0 & 0
\end{array}\right]\left[\begin{array}{c}
\mathbf{u}_{I} \\
p_{I} \\
\mathbf{u}_{\Delta} \\
\mathbf{u}_{\Pi} \\
p_{\Gamma} \\
\lambda_{\Delta}
\end{array}\right]=\left[\begin{array}{c}
\mathbf{f}_{I} \\
0 \\
\mathbf{f}_{\Delta} \\
\mathbf{f}_{\Pi} \\
0 \\
0
\end{array}\right],
$$

where $B_{\Delta}=\left[\begin{array}{llll}B_{\Delta}^{(1)} & B_{\Delta}^{(2)} & \ldots & B_{\Delta}^{(N)}\end{array}\right]$ is a Boolean matrix which enforces the continuity constraint $B_{\Delta} \mathbf{u}_{\Delta}=0$ for the dual displacement degrees of freedom $\mathbf{u}_{\Delta}$ shared by neighboring subdomains. The resulting, leading 4 -by- 4 principal minor will be denoted by $\widetilde{A}$ while the subdomain matrix $A^{(i)}$ denotes the 3 -by-3 block matrix without any Lamé parameter, i.e.,

$$
\widetilde{A}=\left[\begin{array}{cccc}
\mu A_{I I} & B_{I I}^{T} & \mu A_{I \Delta} & \mu A_{I \Pi} \\
B_{I I} & -\frac{1}{\lambda} C_{I I} & B_{I \Delta} & B_{I \Pi} \\
\mu A_{\Delta I} & B_{I \Delta}^{T} & \mu A_{\Delta \Delta} & \mu A_{\Delta \Pi} \\
\mu A_{\Pi I} & B_{I \Pi}^{T} & \mu A_{\Pi \Delta} & \mu A_{\Pi \Pi}
\end{array}\right] \text { and } A^{(i)}=\left[\begin{array}{ccc}
A_{I I}^{(i)} & A_{I \Delta}^{(i)} & A_{I \Pi}^{(i)} \\
A_{\Delta I}^{(i)} & A_{\Delta \Delta}^{(i)} & A_{\Delta \Pi}^{(i)} \\
A_{\Pi I}^{(i)} & A_{\Pi \Delta}^{(i)} & A_{\Pi \Pi}^{(i)}
\end{array}\right]
$$

If we confine ourselves to the case where $\lambda_{\Delta}$ belongs to the range of $B_{\Delta}$, this matrix, although indefinite, is nonsingular under the condition that the primal space is large enough; this simplifies the theory in comparison with that of [44]. We also need to show that the leading principal minors are nonsingular and we can use Sylvester's law of inertia for this purpose. We first look at the subdomain matrices $A^{(i)}$ related to the displacements only; they are positive semi-definite with rigid body motions spanning their null spaces, if any. What is needed is to have enough primal constraints to control the rigid body motions of each subdomain and we also need to use the boundary conditions on $\partial \Omega_{D}$ to be able to conclude that the partially subassembled stiffness matrix, obtained from the $\mu_{i} A^{(i)}$ matrices, is nonsingular. We can then conclude that we do not need to work with null spaces as in [44] for the Stokes problem.

If the primal space is rich enough to constrain the rigid body motions, we can conclude that the leading 3 -by-3 block matrix of (4.1) is nonsingular. This matrix can also be written as a direct sum of subdomain matrices, a fact that, of course, is computationally very attractive. The 4 -by-4 leading block matrix, $\widetilde{A}$, can also be shown to be nonsingular, straightforwardly, since the submatrix containing only multiples of the $A$ sub-matrices is positive definite and so is $C_{I I}$. The non-singularity of the entire matrix follows using similar arguments. 
4.2. The FETI-DP/BDDC reduced system. We will reduce the indefinite system (4.1) to a symmetric, positive definite system by eliminating the $\mathbf{u}_{I}, p_{I}, \mathbf{u}_{\Delta}$, and $\mathbf{u}_{\Pi}$ variables and changing the sign. We obtain a Schur complement $G$ and the reduced linear system

$$
G\left[\begin{array}{c}
p_{\Gamma} \\
\lambda_{\Delta}
\end{array}\right]=g
$$

where

$$
\begin{gathered}
G:=\widetilde{B}_{C} \widetilde{A}^{-1} \widetilde{B}_{C}^{T}+\frac{1}{\lambda} \widetilde{C}, \quad g:=-\widetilde{B}_{C} \widetilde{A}^{-1}\left[\begin{array}{c}
\mathbf{f}_{I} \\
0 \\
\mathbf{f}_{\Delta} \\
\mathbf{f}_{\Pi}
\end{array}\right], \\
\widetilde{B}_{C}:=\left[\begin{array}{cccc}
B_{\Gamma I} & -\frac{1}{\lambda} C_{\Gamma I} & B_{\Gamma \Delta} & B_{\Gamma \Pi} \\
0 & 0 & B_{\Delta} & 0
\end{array}\right] \quad \text { and } \widetilde{C}:=\left[\begin{array}{cc}
C_{\Gamma \Gamma} & 0 \\
0 & 0
\end{array}\right] .
\end{gathered}
$$

As in [44, Section 4], we can prove that $G$ is symmetric, positive definite (positive semidefinite in the Stokes case) by using Sylvester's law of inertia. We also see that the following block-Cholesky factorization reveals $-G$ as the Schur complement of (4.1) with respect to the $\widetilde{C}$ block:

$$
\left[\begin{array}{cc}
I & 0 \\
-\widetilde{B}_{C} \widetilde{A}^{-1} & I
\end{array}\right]\left[\begin{array}{cc}
\widetilde{A} & \widetilde{B}_{C}^{T} \\
\widetilde{B}_{C} & -\frac{1}{\lambda} \widetilde{C}
\end{array}\right]\left[\begin{array}{cc}
I & -\widetilde{A}^{-1} \widetilde{B}_{C}^{T} \\
0 & I
\end{array}\right]=\left[\begin{array}{cc}
\widetilde{A} & 0 \\
0 & -G
\end{array}\right] .
$$

The action of $G$ on given vectors and the construction of the right-hand side $g$ of the reduced system (4.3) require us to compute the action of $\widetilde{A}^{-1}$ on vectors. As shown in [44, Section 4] and below, this can be done, for any vector, by solving a coarse problem associated with the primal variables once and independent subdomain saddle point problems with Neumann boundary conditions, for $\mathbf{u}_{\Delta}$, once. Indeed, partitioning $\widetilde{A}:=\left[\begin{array}{cc}A_{r r} & A_{\Pi r}^{T} \\ A_{\Pi r} & A_{\Pi \Pi}\end{array}\right]$, into interior-dual and primal blocks where

$$
A_{r r}:=\left[\begin{array}{ccc}
\mu A_{I I} & B_{I I}^{T} & \mu A_{I \Delta} \\
B_{I I} & -\frac{1}{\lambda} C_{I I} & B_{I \Delta} \\
\mu A_{\Delta I} & B_{I \Delta}^{T} & \mu A_{\Delta \Delta}
\end{array}\right], \quad A_{\Pi r}:=\left[\begin{array}{lll}
\mu A_{\Pi I} & B_{I \Pi}^{T} & \mu A_{\Pi \Delta}
\end{array}\right],
$$

and defining the primal Schur complement by $S_{\Pi \Pi}:=\mu\left(A_{\Pi \Pi}-A_{\Pi r} A_{r r}^{-1} A_{\Pi r}^{T}\right)$, we see that the action of $\widetilde{A}^{-1}$ can be computed as

$$
\left[\begin{array}{cc}
A_{r r} & A_{\Pi r}^{T} \\
A_{\Pi r} & A_{\Pi \Pi}^{T}
\end{array}\right]^{-1}\left[\begin{array}{c}
\boldsymbol{f}_{r} \\
\boldsymbol{f}_{\Pi}
\end{array}\right]=\left[\begin{array}{c}
A_{r r}^{-1} \boldsymbol{f}_{r}-A_{r r}^{-1} A_{\Pi r}^{T} S_{\Pi \Pi}^{-1}\left(\boldsymbol{f}_{\Pi}-A_{\Pi r} A_{r r}^{-1} \boldsymbol{f}_{r}\right) \\
S_{\Pi \Pi}^{-1}\left(\boldsymbol{f}_{\Pi}-A_{\Pi r} A_{r r}^{-1} \boldsymbol{f}_{r}\right)
\end{array}\right] .
$$

The action of $S_{\Pi}^{-1}$ requires one solution of a coarse problem associated with the primal variables, while the action of $A_{r r}^{-1}$ requires the solution of independent subdomain saddle point problems with Neumann boundary conditions for the dual variables; this last action has to be computed once on $\boldsymbol{f}_{r}$ and once for the second term of the first component of (4.5). The contribution of the latter term can be obtained as a by-product of the computation of the coarse problem matrix $S_{\Pi \Pi \text {; }}$ in a practical algorithm, the action of this second solve is thus replaced by a matrix-vector product. 
We note that during the iteration, we will always solve the first four block equations exactly. We can therefore carry out our analysis in a subspace $\widehat{V}_{0}$ corresponding to vanishing $\boldsymbol{f}_{I}, \boldsymbol{f}_{\Delta}$, and $\boldsymbol{f}_{\Pi}$ :

$$
\begin{array}{r}
\widehat{V}_{0}:=\left\{\left[\boldsymbol{w}_{I}, q_{I}, \boldsymbol{w}_{\Delta}, \boldsymbol{w}_{\Pi}, q_{\Gamma}, \lambda_{\Delta}\right]^{T}: \mu A_{I I} \boldsymbol{w}_{I}+B_{I I}^{T} q_{I}+\mu A_{I \Delta} \boldsymbol{w}_{\Delta}+\mu A_{I \Pi} \boldsymbol{w}_{\Pi}+B_{\Gamma I}^{T} q_{\Gamma}=0\right. \\
B_{I I} \boldsymbol{w}_{I}-\frac{1}{\lambda} C_{I I} q_{I}+B_{I \Delta} \boldsymbol{w}_{\Delta}+B_{I \Pi} \boldsymbol{w}_{\Pi}-\frac{1}{\lambda} C_{I \Gamma} q_{\Gamma}=0 \\
\mu A_{\Delta I} \boldsymbol{w}_{I}+B_{I \Delta}^{T} q_{I}+\mu A_{\Delta \Delta} \boldsymbol{w}_{\Delta}+\mu A_{\Delta \Pi} \boldsymbol{w}_{\Pi}+B_{\Gamma \Delta}^{T} q_{\Gamma}+B_{\Delta}^{T} \lambda_{\Delta}=0 \\
\left.\mu A_{\Pi I} \boldsymbol{w}_{I}+B_{I \Pi}^{T} q_{I}+\mu A_{\Pi \Delta} \boldsymbol{w}_{\Delta}+\mu A_{\Pi \Pi} \boldsymbol{w}_{\Pi}+B_{\Gamma \Pi}^{T} q_{\Gamma}=0\right\}
\end{array}
$$

Note that, in compact form, for all $\left[\boldsymbol{w}_{I}, q_{I}, \boldsymbol{w}_{\Delta}, \boldsymbol{w}_{\Pi}, q_{\Gamma}, \lambda_{\Delta}\right]^{T} \in \widehat{V}_{0}$, it holds

$$
\forall\left[\boldsymbol{w}_{I}, q_{I}, \boldsymbol{w}_{\Delta}, \boldsymbol{w}_{\Pi}\right]^{T}=-\widetilde{A}^{-1} \widetilde{B}_{C}^{T}\left[\begin{array}{c}
q_{\Gamma} \\
\lambda_{\Delta}
\end{array}\right] .
$$

When working with $\widehat{V}_{0}$, we effectively study the decrease of the error during the iteration; the only non-zero residuals will be for the two final rows of (4.1).

4.3. Preliminary results. Using the notation $\boldsymbol{w}=\left[\boldsymbol{w}_{I}, \boldsymbol{w}_{\Delta}, \boldsymbol{w}_{\Pi}\right]^{T}, q=\left[\begin{array}{c}q_{I} \\ q_{\Gamma}\end{array}\right]$, and $\boldsymbol{v}:=\left[\boldsymbol{w}_{I}, q_{I}, \boldsymbol{w}_{\Delta}, \boldsymbol{w}_{\Pi}\right]^{T}$, we will modify the discussion at the beginning of [44, Section 5]. We find by a direct computation that for all $\left[\boldsymbol{w}, q, \lambda_{\Delta}\right]^{T} \in \widehat{V}_{0}$,

$$
\boldsymbol{v}^{T} \widetilde{A} \boldsymbol{v}=\boldsymbol{w}^{T}(\mu A) \boldsymbol{w}+q^{T}\left(\frac{1}{\lambda} C\right) q-q_{\Gamma}^{T}\left(\frac{1}{\lambda} C_{\Gamma \Gamma}\right) q_{\Gamma} .
$$

Thus, we obtain the following two expressions

$$
\boldsymbol{v}^{T} \widetilde{A} \boldsymbol{v}+q_{\Gamma}^{T}\left(\frac{1}{\lambda} C_{\Gamma \Gamma}\right) q_{\Gamma}=\boldsymbol{w}^{T}(\mu A) \boldsymbol{w}+q^{T}\left(\frac{1}{\lambda} C\right) q,
$$

which both define an appropriate norm for the system considered.

Lemma 4.1. Let $\left[\boldsymbol{v}, q_{\Gamma}, \lambda_{\Delta}\right]^{T} \in \widehat{V}_{0}$ and $\boldsymbol{x}=\left[\begin{array}{c}q_{\Gamma} \\ \lambda_{\Delta}\end{array}\right]$. Then

$$
\boldsymbol{x}^{T} G \boldsymbol{x}=\boldsymbol{w}^{T}(\mu A) \boldsymbol{w}+q^{T}\left(\frac{1}{\lambda} C\right) q .
$$

Proof. Since $\left[\boldsymbol{v}, q_{\Gamma}, \lambda_{\Delta}\right]^{T} \in \widehat{V}_{0}$, it holds $\boldsymbol{v}=-\widetilde{A}^{-1} \widetilde{B}_{C}^{T} \boldsymbol{x}$. Hence,

$$
\begin{aligned}
\boldsymbol{x}^{T} G \boldsymbol{x} & =\boldsymbol{x}^{T} \widetilde{B}_{C} \widetilde{A}^{-1} \widetilde{B}_{C}^{T} \boldsymbol{x}+\boldsymbol{x}^{T}\left(\frac{1}{\lambda} \widetilde{C}\right) \boldsymbol{x} \\
& =\left(\widetilde{A}^{-1} \widetilde{B}_{C}^{T} \boldsymbol{x}\right)^{T} \widetilde{A} \widetilde{A}^{-1} \widetilde{B}_{C}^{T} \boldsymbol{x}+q_{\Gamma}^{T}\left(\frac{1}{\lambda} C_{\Gamma \Gamma}\right) q_{\Gamma} \\
& =\boldsymbol{v}^{T} \widetilde{A} \boldsymbol{v}+q_{\Gamma}^{T}\left(\frac{1}{\lambda} C_{\Gamma \Gamma}\right) q_{\Gamma}=\boldsymbol{w}^{T}(\mu A) \boldsymbol{w}+q^{T}\left(\frac{1}{\lambda} C\right) q .
\end{aligned}
$$

Let us now define the matrices

$$
\widetilde{B}:=\left[\begin{array}{ccc}
B_{I I} & B_{I \Delta} & B_{I \Pi} \\
B_{\Gamma I} & B_{\Gamma \Delta} & B_{\Gamma \Pi}
\end{array}\right], \quad \widetilde{B}^{(i)}:=\left[\begin{array}{ccc}
B_{I I}^{(i)} & B_{I \Delta}^{(i)} & B_{I \Pi}^{(i)} \\
B_{\Gamma I}^{(i)} & B_{\Gamma \Delta}^{(i)} & B_{\Gamma \Pi}^{(i)}
\end{array}\right] .
$$

The following two lemmas hold: 
LeMma 4.2. For any $\boldsymbol{w}_{i}=\left[\boldsymbol{w}_{i I}, \boldsymbol{w}_{i \Delta}, \boldsymbol{w}_{i \Pi}\right]^{T}$ and $q_{i} \in Q_{i}$, it holds

$$
q_{i}^{T} \widetilde{B}^{(i)} \boldsymbol{w}_{i} \leq \sqrt{3 / 2}\left(\boldsymbol{w}_{i}^{T} A^{(i)} \boldsymbol{w}_{i}\right)^{1 / 2}\left(q_{i}^{T} C^{(i)} q_{i}\right)^{1 / 2} .
$$

This results from the discrete version of the bound

$$
\int_{\Omega_{i}} \operatorname{div}(\boldsymbol{v}) \operatorname{div}(\boldsymbol{v}) d x \leq 3 \int_{\Omega_{i}} \varepsilon(\boldsymbol{v}): \varepsilon(\boldsymbol{v}) d x=3 / 2 a_{i}(\boldsymbol{v}, \boldsymbol{v}),
$$

which implies $\left|b_{i}(\boldsymbol{v}, q)\right|=\left|-\int_{\Omega_{i}} \operatorname{div}(\boldsymbol{v}) q d x\right| \leq \sqrt{3 / 2} a_{i}(\boldsymbol{v}, \boldsymbol{v})^{1 / 2} c_{i}(q, q)^{1 / 2}$.

The following lemma is basically a generalization of [20, Lemma 2.3].

Lemma 4.3. Let $\left[\begin{array}{c}\boldsymbol{w} \\ q\end{array}\right]$ satisfy $\left[\begin{array}{cc}\mu A & B^{T} \\ B & -\frac{1}{\lambda} C\end{array}\right]\left[\begin{array}{l}\boldsymbol{w} \\ q\end{array}\right]=\left[\begin{array}{l}\boldsymbol{f} \\ g\end{array}\right]$. Then, the following bound holds:

$$
\left(\boldsymbol{w}^{T}(\mu A) \boldsymbol{w}\right)+q^{T}\left(\frac{1}{\lambda} C\right) q \leq 4 f^{T}(\mu A)^{-1} f+\frac{2}{\beta^{2}} g^{T}\left(\frac{1}{\mu} C\right)^{-1} g,
$$

where $\beta^{2}$ is an inf-sup constant such that $q^{T} B(\mu A)^{-1} B^{T} q \geq \beta^{2} q^{T}\left(\frac{1}{\mu} C\right) q, \quad \forall q$.

Proof. By using a formula for the inverse of the matrix, we find that

$$
(\mu A)^{1 / 2} \boldsymbol{w}=\left(I-(\mu A)^{-1 / 2} B^{T} S^{-1} B(\mu A)^{-1 / 2}\right)(\mu A)^{-1 / 2} f+(\mu A)^{-1 / 2} B^{T} S^{-1} g,
$$

where $S:=B(\mu A)^{-1} B^{T}+\frac{1}{\lambda} C$. Since $S^{-1} \leq\left(B(\mu A)^{-1} B^{T}\right)^{-1}$, we find that

$$
\left.(\mu A)^{-1 / 2} B^{T} S^{-1} B(\mu A)^{-1 / 2}\right) \leq(\mu A)^{-1 / 2} B^{T}\left(B(\mu A) B^{T}\right)^{-1} B(\mu A)^{-1 / 2} \leq I
$$

since $(\mu A)^{-1 / 2} B^{T}\left(B(\mu A) B^{T}\right)^{-1} B(\mu A)^{-1 / 2}$ is a projection. Therefore, the $\ell_{2}-$ norm of the first term on the right-hand side of (4.10) is bounded by $\left\|(\mu A)^{-1 / 2} f\right\|_{\ell_{2}}$.

Similarly, we find that

$$
\left(\frac{1}{\lambda} C\right)^{1 / 2} q=\left(\frac{1}{\lambda} C\right)^{1 / 2} S^{-1} B(\mu A)^{-1} f-\left(\frac{1}{\lambda} C\right)^{1 / 2} S^{-1} g .
$$

The square of the $\ell_{2}-$ norm of its first term can be estimated as follows:

$$
\begin{gathered}
f^{T}(\mu A)^{-1} B^{T} S^{-1}\left(\frac{1}{\lambda} C\right) S^{-1} B(\mu A)^{-1} f \leq f^{T}(\mu A)^{-1} B^{T} S^{-1} S S^{-1} B(\mu A)^{-1} f \leq \\
f^{T}(\mu A)^{-1 / 2}\left((\mu A)^{-1 / 2} B^{T}\left(B(\mu A)^{-1} B^{T}\right)^{-1} B(\mu A)^{-1 / 2}\right)(\mu A)^{-1 / 2} f \leq f^{T}(\mu A)^{-1} f,
\end{gathered}
$$

by again using (4.11).

Combining the two quadratic forms that operate on $g$, we find that the sum of those two terms equals $g^{T} S^{-1} g$, which can be estimated from above by $\frac{1}{\beta^{2}} g^{T}\left(\frac{1}{\mu} C\right)^{-1} g$ since $S=B(\mu A)^{-1} B^{T}+\frac{1}{\lambda} C \geq \beta^{2}\left(\frac{1}{\mu} C\right)$. The proof can now be easily completed. $\square$

5. The block FETI-DP/BDDC preconditioner. In order to develop our theory, we consider the isogeometric block FETI-DP/BDDC preconditioner

$$
M^{-1}=\left[\begin{array}{cc}
M_{p_{\Gamma}}^{-1} & 0 \\
0 & M_{\lambda_{\Delta}}^{-1}
\end{array}\right] .
$$

for the reduced system (4.2) with the coefficient matrix

$$
G=\left[\begin{array}{ll}
G_{p_{\Gamma} p_{\Gamma}} & G_{\lambda_{\Delta} p_{\Gamma}}^{T} \\
G_{\lambda_{\Delta} p_{\Gamma}} & G_{\lambda_{\Delta} \lambda_{\Delta}}
\end{array}\right]
$$


In a practical algorithm, we can also consider block-triangular preconditioning variants to accelerate a GMRES solver, and exploit robust and possibly iterative sub-solvers, built on top of the individual preconditioners introduced in Sections 5.2 and 5.3, and embedded in a flexible outer Krylov method.

We first review the construction of a deluxe scaling algorithm (see Dohrmann and Widlund [14]), as it is crucial to obtain a robust solver for our isogeometric problem given in (2.7), see also Section 7.1, and which we have found to be superior to the more common $\rho$ - and stiffness-scalings, in our previous studies on isogeometric solvers, see $[6,7,37]$.

5.1. Deluxe scaling. In BDDC and FETI-DP methods, the average $\overline{\boldsymbol{w}}:=E_{D} \boldsymbol{w}$ of an element in the partially continuous space is computed separately for the sets of interface degrees of freedom of vertex, edge, and face equivalence classes. We start by defining the deluxe scaling in the simplest case of a class $\mathcal{F}$ with only two elements, $i, j$, as for an edge in two dimensions or a face in three dimensions; for more details on the fat interface and the definition of the fat equivalence classes, we refer to [6, Section 4.2] and [7, Section 3]. Let $S^{(i)}$ be the subdomain interface Schur complement of the matrix $\mu_{i} A^{(i)}$ associated with $\Omega_{i}$, and define two principal minors, $S_{\mathcal{F}}^{(i)}$ and $S_{\mathcal{F}}^{(j)}$, obtained from $S^{(i)}$ and $S^{(j)}$ by removing all rows and columns which do not belong to $\mathcal{F}$.

Let $\boldsymbol{w}_{\mathcal{F}}^{(i)}$ denote the restriction of an element in the dual space to the face $\mathcal{F}$. The deluxe average across $\mathcal{F}$ is then defined as

$$
\overline{\boldsymbol{w}}_{\mathcal{F}}=\left(S_{\mathcal{F}}^{(i)}+S_{\mathcal{F}}^{(j)}\right)^{-1}\left(S_{\mathcal{F}}^{(i)} \boldsymbol{w}_{\mathcal{F}}^{(i)}+S_{\mathcal{F}}^{(j)} \boldsymbol{w}_{\mathcal{F}}^{(j)}\right) .
$$

In three dimensions, we also need to define deluxe averaging operators for subdomain edges and subdomain vertices. Given the simple hexahedral subdomain geometry of the parameter space that we are considering, we find that in all these cases the equivalence classes will have four and eight elements for any fat subdomain edge and vertex, respectively, in the interior of $\Omega$. Thus, for a fat subdomain edge $\mathcal{E}$ shared by subdomains $\Omega_{i}, \Omega_{j}, \Omega_{k}$, and $\Omega_{\ell}$, we use the formula

$$
\overline{\boldsymbol{w}}_{\mathcal{E}}:=\left(S_{\mathcal{E}}^{(i)}+S_{\mathcal{E}}^{(j)}+S_{\mathcal{E}}^{(k)}+S_{\mathcal{E}}^{(\ell)}\right)^{-1}\left(S_{\mathcal{E}}^{(i)} \boldsymbol{w}_{\mathcal{E}}^{(i)}+S_{\mathcal{E}}^{(j)} \boldsymbol{w}_{\mathcal{E}}^{(j)}+S_{\mathcal{E}}^{(k)} \boldsymbol{w}_{\mathcal{E}}^{(k)}+S_{\mathcal{E}}^{(\ell)} \boldsymbol{w}_{\mathcal{E}}^{(\ell)}\right)
$$

An analogous formula holds for the fat vertices and involves eight operators. Edges and vertices located on the Neumann boundary of the domain will have fewer elements, depending on the number of subdomain boundaries that share them.

For each subdomain $\Omega_{i}$, we then define a scaling matrix by its restriction $D_{\Delta}^{(i)}$ to subdomain $\Omega_{i}$ as the direct sum of diagonal blocks given by the deluxe scaling of the face, edge, and vertex terms belonging to the interface of $\Omega_{i}$ :

- for subdomain faces: $\quad D_{\mathcal{F}}^{(i)}:=S_{\mathcal{F}}^{(i)}\left(S_{\mathcal{F}}^{(i)}+S_{\mathcal{F}}^{(j)}\right)^{-1}$

- for subdomain edges: $\quad D_{\mathcal{E}}^{(i)}:=S_{\mathcal{E}}^{(i)}\left(S_{\mathcal{E}}^{(i)}+S_{\mathcal{E}}^{(j)}+S_{\mathcal{E}}^{(k)}+S_{\mathcal{E}}^{(\ell)}\right)^{-1}$,

- for subdomain vertices: an analogous formula with eight operators.

For the use of these operators, see Subsection 5.3. In terms of the complementary projection operator $P_{D}=I-E_{D}$, we find, for a fat face of $\Omega_{i}$, that

$$
P_{D} \boldsymbol{w}_{\mathcal{F}}=\left(S_{\mathcal{F}}^{(i)}+S_{\mathcal{F}}^{(j)}\right)^{-1} S_{\mathcal{F}}^{(j)}\left(\boldsymbol{w}_{\mathcal{F}}^{(i)}-\boldsymbol{w}_{\mathcal{F}}^{(j)}\right),
$$


and for a fat edge of $\Omega_{i}, P_{D} \boldsymbol{w}_{\mathcal{E}}=$

$$
\left(S_{\mathcal{E}}^{(i)}+S_{\mathcal{E}}^{(j)}+S_{\mathcal{E}}^{(k)}+S_{\mathcal{E}}^{(\ell)}\right)^{-1}\left(S_{\mathcal{E}}^{(j)}\left(\boldsymbol{w}_{\mathcal{E}}^{(i)}-\boldsymbol{w}_{\mathcal{E}}^{(j)}\right)+S_{\mathcal{E}}^{(k)}\left(\boldsymbol{w}_{\mathcal{E}}^{(i)}-\boldsymbol{w}_{\mathcal{E}}^{(k)}\right)+S_{\mathcal{E}}^{(\ell)}\left(\boldsymbol{w}_{\mathcal{E}}^{(i)}-\boldsymbol{w}_{\mathcal{E}}^{(\ell)}\right)\right),
$$

and analogously with eight operators for a fat vertex; see also [6, Section 4.2].

5.2. The pressure sub-solver. In the analysis of our algorithm, the pressure sub-solver $M_{p_{\Gamma}}^{-1}$ is chosen as the inverse of $\frac{1}{\mu} S_{\Gamma \Gamma}^{C}$ obtained from the subdomain mass matrices and associated with the interface pressure variables $p_{\Gamma}$. This matrix is obtained by sub-assembling the local Schur complements $S_{\Gamma \Gamma}^{C^{(i)}}$ of the subdomain mass matrices $C^{(i)}$ weighted by $\frac{1}{\mu_{i}}$ and defined by

$$
\frac{1}{\mu_{i}} S_{\Gamma \Gamma}^{C^{(i)}}:=\frac{1}{\mu_{i}}\left(C_{\Gamma \Gamma}^{(i)}-C_{\Gamma I}^{(i)} C_{I I}^{(i)-1} C_{I \Gamma}^{(i)}\right) .
$$

To develop a competitive algorithm, we then replace the inverse of this Schur complement, defining $M_{p_{\Gamma}}^{-1}$, by a preconditioner, namely a BDDC deluxe variant built from the subdomain matrices $\frac{1}{\mu_{i}} S_{\Gamma \Gamma}^{C^{(i)}}$. In our experience, we do not need any primal subspace for this preconditioner of the $p_{\Gamma}$ variable.

5.3. The Lagrange multiplier sub-solver. The preconditioner $M_{\lambda_{\Delta}}^{-1}$ for the $\lambda_{\Delta}$ variable is the FETI-DP preconditioner

$$
M_{\lambda_{\Delta}}^{-1}=B_{\Delta, D} H_{\Delta} B_{\Delta, D}^{T},
$$

where $H_{\Delta}$ is the direct sum of the local discrete harmonic extension operators $H_{\Delta}^{(i)}$, $i=1, \ldots, N$, each defined by a local elliptic problem on each of the subdomains $\Omega_{i}$ :

$$
\mu_{i}\left[\begin{array}{cc}
A_{I I}^{(i)} & A_{I \Delta}^{(i)} \\
A_{\Delta I}^{(i)} & A_{\Delta \Delta}^{(i)}
\end{array}\right]\left[\begin{array}{c}
\mathbf{u}_{I}^{(i)} \\
\mathbf{u}_{\Delta}^{(i)}
\end{array}\right]=\left[\begin{array}{c}
0 \\
H_{\Delta}^{(i)} \mathbf{u}_{\Delta}^{(i)}
\end{array}\right],
$$

with given boundary displacement $\mathbf{u}_{\Delta}^{(i)}$ and zero primal displacement, i.e., $\mathbf{u}_{\Pi}^{(i)}=0$. Using linearly independent primal constraints and non-redundant multipliers, the scaling operator $B_{\triangle, D}$ can be written as in [28, Section 4, p. 65]

$$
B_{\Delta, D}=\left(B_{\Delta} D_{\Delta}^{-1} B_{\Delta}^{T}\right)^{-1} B_{\Delta} D_{\Delta}^{-1}
$$

where $D_{\Delta}$ is a diagonal, or block diagonal scaling matrix in the deluxe case, built from the subdomain matrices $H_{\Delta}^{(i)}$. We note that $H_{\Delta}^{(i)}$ is a Schur complement of the matrix on the left hand side of (5.4).

5.4. The choice of the primal constraints. As in our previous work on isogeometric solvers for compressible elasticity [37, Section 5.2], we can consider a number of choices of primal spaces of increasing dimension, employing different combinations of fat or thin vertex, edge, and face constraints. Constraints can also be built from rigid body modes by restricting the vectors that represent them to each relevant vertex, edge, or face equivalence class and running a singular value decomposition (SVD) on these sets of subvectors, in order to eliminate any possible null rotation or linear dependence. We refer to [37, Section 5.2] for more details and to Section 7 for the specific primal choices used in our numerical tests. Our previous paper contains two main results, Theorems 4.1 and 4.2 , which provides relatively small primal spaces which guarantee bounds of the condition numbers of the form $C(1+\log (H / h))^{3}$ and $C(1+\log (H / h))^{2}$, respectively. 
6. Convergence rate analysis. The main result of this paper is a bound on the condition number of the preconditioned operator $M^{-1} G$, given below in Theorem 6.1. We will need to show that we can work out a proof in the presence of the $C$ matrices. Of particular importance in the Tu-Li proof is that $\tilde{A}^{-1} \widetilde{B}_{C}^{T} \boldsymbol{x} \in \tilde{V}_{0}$; see [44, Section 5] for the definition of this space. In the Stokes case, this easily follows by applying the second row of the matrix of (4.1) to this expression and noticing that a certain block matrix of $\widetilde{B}_{C}^{T}$ equals 0 . This is not the case for almost incompressible elasticity and therefore, we will approach the proof of our main result differently.

In Lemmas 6.2 and 6.3, we will prove upper and lower bounds of $\boldsymbol{x}^{T} G M^{-1} G \boldsymbol{x}$ in terms $\boldsymbol{x}^{T} G \boldsymbol{x}$. For this, we will use Lemma 4.1, which states that the energy of the system can be written as $\boldsymbol{x}^{T} G \boldsymbol{x}$ for elements in the space $\widehat{V}_{0}$. These bounds will provide an upper and a lower bound for the eigenvalues of $M^{-1} G$ in terms of an infsup parameter and condition number of a related algorithm for compressible elasticity. We can then use our results in [37] to obtain bounds with two or three logarithmic factors, respectively; see [37, Th. 4.1 and 4.2]. A bound for the condition number of $M^{-1} G$ can be expressed in terms of the bounds developed in these two lemmas.

6.1. The main result. The condition number of the preconditioned operator $M^{-1} G$ can be bounded as follows.

THEOREM 6.1. $\forall \boldsymbol{x}=\left[\begin{array}{c}p_{\Gamma} \\ \lambda_{\Delta}\end{array}\right]$, it holds

$$
C(\beta)^{-1} \boldsymbol{x}^{T} G \boldsymbol{x} \leq \boldsymbol{x}^{T} G M^{-1} G \boldsymbol{x} \leq(3+\Phi(H / h)) \boldsymbol{x}^{T} G \boldsymbol{x},
$$

where $C(\beta):=\left(10+\frac{12}{\beta^{2}}\right), \beta$ the inf-sup parameter of the fully assembled saddlepoint system, and $\Phi(H / h)$ is the condition number of a related compressible elasticity problem. Therefore,

$$
\kappa\left(M^{-1} G\right) \leq C(\beta)(3+\Phi(H / h))
$$

6.2. Proof of the upper bound. We will now prove a counterpart to [44, Lemma 7.2]. We will use the same notation as in subsection 4.3.

$$
\begin{aligned}
& \text { Lemma } 6.2 . \forall \boldsymbol{y}=G \boldsymbol{x} \text {, let }\left[\boldsymbol{u}, p, \lambda_{\Delta}\right]^{T}=\left[\boldsymbol{v}, p_{\Gamma}, \lambda_{\Delta}\right]^{T} \in \widehat{V}_{0}, \text { satisfy } \\
& \widetilde{B}_{C} \boldsymbol{v}-\frac{1}{\lambda} \widetilde{C}\left[\begin{array}{c}
p_{\Gamma} \\
\lambda_{\Delta}
\end{array}\right]=\text { Gx. Then } \\
& \boldsymbol{x}^{T} G M^{-1} G \boldsymbol{x} \leq(3+\Phi(H / h))\left(\boldsymbol{u}^{T}(\mu A) \boldsymbol{u}+2 \max _{i}\left(\frac{\mu_{i}}{\lambda_{i}}\right) p^{T}\left(\frac{1}{\lambda} C\right) p\right) .
\end{aligned}
$$

Proof. The existence of $\left[\boldsymbol{v}, p_{\Gamma}, \lambda_{\Delta}\right]^{T} \in \widehat{V}_{0}$, satisfying $\widetilde{B}_{C} \boldsymbol{v}-\frac{1}{\lambda} \widetilde{C}\left[\begin{array}{c}p_{\Gamma} \\ \lambda_{\Delta}\end{array}\right]=G \boldsymbol{x}$, follows directly from the definition of $\widehat{V}_{0}$. We then write the left-hand side of (6.1) as

$$
\left(\widetilde{B}_{C} \boldsymbol{v}-\frac{1}{\lambda} \widetilde{C}\left[\begin{array}{c}
p_{\Gamma} \\
\lambda_{\Delta}
\end{array}\right]\right)^{T} M^{-1}\left(\widetilde{B}_{C} \boldsymbol{v}-\frac{1}{\lambda} \widetilde{C}\left[\begin{array}{c}
p_{\Gamma} \\
\lambda_{\Delta}
\end{array}\right]\right)
$$

and we will prove an upper bound of this expression in terms of the energy of the system given in terms of $\boldsymbol{u}^{T}(\mu A) \boldsymbol{u}$ and $p^{T}\left(\frac{1}{\lambda} C\right) p$. 
Given $\left[\boldsymbol{u}, p, \lambda_{\Delta}\right]^{T}=\left[\boldsymbol{u}_{I}, p_{I}, \boldsymbol{u}_{\Delta}, \boldsymbol{u}_{\Pi}, p_{\Gamma}, \lambda_{\Delta}\right]^{T} \in \widehat{V}_{0}$, consider the first of the two components of $\widetilde{B}_{C} \boldsymbol{v}-\frac{1}{\lambda} \widetilde{C}\left[\begin{array}{c}p_{\Gamma} \\ \lambda_{\Delta}\end{array}\right]$, namely,

$$
g_{p_{\Gamma}}:=B_{\Gamma I} \boldsymbol{u}_{I}-1 / \lambda C_{\Gamma I} p_{I}+B_{\Gamma \Delta} \boldsymbol{u}_{\Delta}+B_{\Gamma \Pi} \boldsymbol{u}_{\Pi}-1 / \lambda C_{\Gamma \Gamma} p_{\Gamma} .
$$

Since $\left[\boldsymbol{u}, p, \lambda_{\Delta}\right]^{T} \in \widehat{V}_{0}$, we also have $B_{I I} \boldsymbol{u}_{I}-\frac{1}{\lambda} C_{I I} p_{I}+B_{I \Delta} \boldsymbol{u}_{\Delta}+B_{I \Pi} \boldsymbol{u}_{\Pi}-\frac{1}{\lambda} C_{I \Gamma} p_{\Gamma}=0$.

The preconditioner for this first component is given by $\frac{1}{\mu} S_{\Gamma \Gamma}^{C}$. But we can instead work with $\frac{1}{\mu} C$ and let its inverse act on $\widetilde{B} \boldsymbol{u}-\frac{1}{\lambda} C p$ since the first row of that matrix vanishes.

We then find that

$$
\begin{aligned}
g_{p_{\Gamma}}^{T}\left(\frac{1}{\mu} S_{\Gamma \Gamma}^{C}\right)^{-1} g_{p_{\Gamma}} & =\left(\widetilde{B} \mathbf{u}-\frac{1}{\lambda} C p\right)^{T}\left(\frac{1}{\mu} C\right)^{-1}\left(\widetilde{B} \mathbf{u}-\frac{1}{\lambda} C p\right) \\
& \leq 2(\widetilde{B} \mathbf{u})^{T}\left(\frac{1}{\mu} C\right)^{-1}(\widetilde{B} \mathbf{u})+2\left(\frac{1}{\lambda} C p\right)^{T}\left(\frac{1}{\mu} C\right)^{-1}\left(\frac{1}{\lambda} C p\right)
\end{aligned}
$$

The first term on the right-hand side of (6.2) satisfies

$$
2 \mathbf{u}^{T} \widetilde{B}^{T}\left(\frac{1}{\mu} C\right)^{-1} \widetilde{B} \mathbf{u}=2 \max _{q} \frac{\left((\widetilde{B} \mathbf{u})^{T} q\right)^{2}}{q^{T}\left(\frac{1}{\mu} C\right) q}=2 \max _{q} \frac{\left(\sum_{i}\left(\widetilde{B}^{(i)} \mathbf{u}_{i}\right)^{T} q_{i}\right)^{2}}{\sum_{i} q_{i}^{T} \frac{1}{\mu_{i}} C^{(i)} q_{i}} .
$$

By Lemma 4.2, the terms in the numerator can be bounded by

$$
\left|\left(\widetilde{B}^{(i)} \mathbf{u}_{i}\right)^{T} q_{i}\right| \leq \sqrt{\frac{3}{2}\left(\mathbf{u}_{i}^{T} A^{(i)} \mathbf{u}_{i}\right)\left(q_{i}^{T} C^{(i)} q_{i}\right)}=\sqrt{\frac{3}{2}\left(\mathbf{u}_{i}^{T}\left(\mu_{i} A^{(i)}\right) \mathbf{u}_{i}\right)\left(q_{i}^{T}\left(\frac{1}{\mu_{i}} C^{(i)}\right) q_{i}\right)}
$$

and we have

$$
2 \mathbf{u}^{T} \widetilde{B}^{T}\left(\frac{1}{\mu} C\right)^{-1} \widetilde{B} \mathbf{u} \leq 3 \mathbf{u}^{T}(\mu A) \mathbf{u} .
$$

The second term on the right-hand side of (6.2) satisfies

$$
\begin{array}{r}
2\left(\frac{1}{\lambda} C p\right)^{T}\left(\frac{1}{\mu} C\right)^{-1}\left(\frac{1}{\lambda} C p\right)=2 \max _{q} \frac{\left(\left(\frac{1}{\lambda} C p\right)^{T} q\right)^{2}}{q^{T}\left(\frac{1}{\mu} C\right) q}=2 \max _{q} \frac{\left(\sum_{i} \frac{1}{\lambda_{i}}\left(C^{(i)} p_{i}\right)^{T} q_{i}\right)^{2}}{\sum_{i} q_{i}^{T} \frac{1}{\mu_{i}} C^{(i)} q_{i}} \leq \\
\leq 2 \max _{q} \frac{\left(\sum_{i} \frac{\mu_{i}}{\lambda_{i}^{2}} p_{i}^{T} C^{(i)} p_{i}\right)\left(\sum_{i} \frac{1}{\mu_{i}} q_{i}^{T} C^{(i)} q_{i}\right)}{\sum_{i} q_{i}^{T} \frac{1}{\mu_{i}} C^{(i)} q_{i}} \leq 2 \max _{i}\left(\frac{\mu_{i}}{\lambda_{i}}\right) p^{T}\left(\frac{1}{\lambda} C\right) p .
\end{array}
$$

The bound related to the second block of $M^{-1}$ can be borrowed directly from work on compressible elasticity. Bounds for $\Phi(H / h)$ are provided in [37].

6.3. Proof of the lower bound. We will now prove a counterpart to [44, Lemma 7.3]. As in the previous subsection, we will use the same notation as in subsection 4.3. By using similar arguments as in the previous subsection, we can provide a lower bound in terms of $\boldsymbol{x}^{T} G \boldsymbol{x}$.

Lemma 6.3. $\forall \boldsymbol{y}=G \boldsymbol{x}:=\left[\begin{array}{c}g_{p_{\Gamma}} \\ g_{\lambda}\end{array}\right] \in\left(Q_{\Gamma} \times \Lambda\right)^{\prime}$, let $\left[\boldsymbol{u}, p, \lambda_{\Delta}\right]^{T}=\left[\boldsymbol{v}, p_{\Gamma}, \lambda_{\Delta}\right]^{T} \in \widehat{V}_{0}$ satisfy $\widetilde{B}_{C} \boldsymbol{v}-\frac{1}{\lambda} \widetilde{C}\left[\begin{array}{c}p_{\Gamma} \\ \lambda_{\Delta}\end{array}\right]=\boldsymbol{y}$. Then,

$$
\boldsymbol{u}^{T}(\mu A) \boldsymbol{u}+p^{T}\left(\frac{1}{\lambda} C\right) p \leq C(\beta) \boldsymbol{y}^{T} M^{-1} \boldsymbol{y}
$$


with $C(\beta)=\left(10+\frac{12}{\beta^{2}}\right)$ where $\beta$ is the inf-sup parameter of the fully assembled system.

Proof. Given $\boldsymbol{y}=\left[\begin{array}{c}g_{p_{\Gamma}} \\ g_{\lambda}\end{array}\right]$, let $\mathbf{u}_{\Delta}^{(1)}=B_{\Delta, D}^{T} g_{\lambda}, \mathbf{u}_{\Pi}^{(1)}=\mathbf{0}, p^{(1)}=0$ and $\lambda_{\Delta}^{(1)}=0$.

On each subdomain $\Omega_{i}$, let $\mathbf{u}_{I}^{(1)}$ be obtained by solving (5.4) on each subdomain. Denote then the corresponding global vectors by $\mathbf{u}^{(1)}$ and $p^{(1)}$. We find that

$$
\widetilde{B}_{C} \boldsymbol{v}^{(1)}=\left[\begin{array}{cccc}
B_{\Gamma I} & -\frac{1}{\lambda} C_{\Gamma I} & B_{\Gamma \Delta} & B_{\Gamma \Pi} \\
0 & 0 & B_{\Delta} & 0
\end{array}\right]\left[\begin{array}{c}
\mathbf{u}_{I}^{(1)} \\
p_{I}^{(1)} \\
\mathbf{u}_{\Delta}^{(1)} \\
\mathbf{u}_{\Pi}^{(1)}
\end{array}\right]=\left[\begin{array}{c}
B_{\Gamma I} \mathbf{u}_{I}^{(1)}+B_{\Gamma \Delta} \mathbf{u}_{\Delta}^{(1)} \\
g_{\lambda}
\end{array}\right],
$$

since $p_{I}^{(1)}=0, \mathbf{u}_{\Pi}^{(1)}=\mathbf{0}$, and $B_{\Delta} \mathbf{u}_{\Delta}^{(1)}=B_{\Delta} B_{\Delta, D}^{T} g_{\lambda}=g_{\lambda}$.

Recall also that $\mathbf{u}^{(1)^{T}}(\mu A) \mathbf{u}^{(1)}=\left|\mathbf{u}_{\Delta}^{(1)}\right|_{H_{\Delta}}^{2}$.

Consider now the solution $\left[\mathbf{u}_{I}^{(2)}, p_{I}^{(2)}, \mathbf{u}_{\Gamma}^{(2)}, p_{\Gamma}^{(2)}\right]^{T}$ of the fully assembled system, as in (3.9),

$$
\left[\begin{array}{cccc}
\mu A_{I I} & B_{I I}^{T} & \mu A_{I \Gamma} & B_{\Gamma I}^{T} \\
B_{I I} & -\frac{1}{\lambda} C_{I I} & B_{I \Gamma} & -\frac{1}{\lambda} C_{I \Gamma} \\
\mu A_{\Gamma I} & B_{I \Gamma}^{T} & \mu A_{\Gamma \Gamma} & B_{\Gamma \Gamma}^{T} \\
B_{\Gamma I} & -\frac{1}{\lambda} C_{\Gamma I} & B_{\Gamma \Gamma} & -\frac{1}{\lambda} C_{\Gamma \Gamma}
\end{array}\right]\left[\begin{array}{c}
\mathbf{u}_{I}^{(2)} \\
p_{I}^{(2)} \\
\mathbf{u}_{\Gamma}^{(2)} \\
p_{\Gamma}^{(2)}
\end{array}\right]=\left[\begin{array}{c}
-\mu A_{I I} \mathbf{u}_{I}^{(1)}-\mu A_{I \Delta} \mathbf{u}_{\Delta}^{(1)} \\
-B_{I I} \mathbf{u}_{I}^{(1)}-B_{I \Delta} \mathbf{u}_{\Delta}^{(1)} \\
-\mu A_{\Gamma I} \mathbf{u}_{I}^{(1)}-\mu A_{\Gamma \Delta} \mathbf{u}_{\Delta}^{(1)} \\
g_{p_{\Gamma}}-B_{\Gamma I} \mathbf{u}_{I}^{(1)}-B_{\Gamma \Delta} \mathbf{u}_{\Delta}^{(1)}
\end{array}\right]
$$

and let $\mathbf{u}^{(2)}=\left[\begin{array}{c}\mathbf{u}_{I}^{(2)} \\ \mathbf{u}_{\Gamma}^{(2)}\end{array}\right]$. We now obtain bounds for $\mathbf{u}^{(2)}$ and $p^{(2)}$ by using (4.9) after extracting $f$ and $g$, the elements of the right-hand side of the system. We also use (6.3) and obtain

$$
\begin{aligned}
& \mathbf{u}^{(2)^{T}}(\mu A) \mathbf{u}^{(2)}+p^{(2)^{T}}\left(\frac{1}{\lambda} C\right) p^{(2)} \leq 4\left(\mu A \mathbf{u}^{(1)}\right)^{T}(\mu A)^{-1}\left(\mu A \mathbf{u}^{(1)}\right)+ \\
& \frac{4}{\beta^{2}}\left(\left(\widetilde{B} \mathbf{u}^{(1)}\right)^{T}\left(\frac{1}{\mu} C\right)^{-1} \widetilde{B} \mathbf{u}^{(1)}+\left[0 g_{p_{\Gamma}}^{T}\right]\left(\frac{1}{\mu} C\right)^{-1}\left[0 g_{p_{\Gamma}}^{T}\right]^{T}\right) \\
& \leq 4\left(\mathbf{u}^{(1)}\right)^{T}(\mu A) \mathbf{u}^{(1)}+\frac{4}{\beta^{2}}\left(\frac{3}{2} \mathbf{u}^{(1)^{T}}(\mu A) \mathbf{u}^{(1)}+g_{p_{\Gamma}}^{T}\left(\frac{1}{\mu} S_{\Gamma \Gamma}^{C}\right)^{-1} g_{p_{\Gamma}}\right) \\
& \leq 4 \mathbf{u}^{(1)^{T}}(\mu A) \mathbf{u}^{(1)}+\frac{6}{\beta^{2}} \mathbf{u}^{(1)^{T}}(\mu A) \mathbf{u}^{(1)}+\frac{4}{\beta^{2}} g_{p_{\Gamma}}^{T}\left(\frac{1}{\mu} S_{\Gamma \Gamma}^{C}\right)^{-1} g_{p_{\Gamma}} \leq\left(4+\frac{6}{\beta^{2}}\right) \boldsymbol{y}^{T} M^{-1} \boldsymbol{y},
\end{aligned}
$$

where we have used the identity

$$
\begin{aligned}
& \boldsymbol{y}^{T} M^{-1} \boldsymbol{y}=\left[g_{p_{\Gamma}}^{T} g_{\lambda}^{T}\right]\left[\begin{array}{cc}
\left(\frac{1}{\mu} S_{\Gamma \Gamma}^{C}\right)^{-1} & 0 \\
0 & M_{\lambda_{\Delta}}^{-1}
\end{array}\right]\left[\begin{array}{c}
g_{p_{\Gamma}} \\
g_{\lambda}
\end{array}\right] \\
& =g_{p_{\Gamma}}^{T}\left(\frac{1}{\mu} S_{\Gamma \Gamma}^{C}\right)^{-1} g_{p_{\Gamma}}+g_{\lambda} M_{\lambda_{\Delta}}^{-1} g_{\lambda}=g_{p_{\Gamma}}^{T}\left(\frac{1}{\mu} S_{\Gamma \Gamma}^{C}\right)^{-1} g_{p_{\Gamma}}+\left|\mathbf{u}_{\Delta}^{(1)}\right|_{H_{\Delta}}^{2} \\
& =g_{p_{\Gamma}}^{T}\left(\frac{1}{\mu} S_{\Gamma \Gamma}^{C}\right)^{-1} g_{p_{\Gamma}}+\mathbf{u}^{(1)^{T}}(\mu A) \mathbf{u}^{(1)} .
\end{aligned}
$$

Here $\beta$ is the inf-sup parameter for the fully assembled saddle-point problem. The contribution from $\boldsymbol{u}^{(1)}$ can be bounded by using that $\mathbf{u}^{(1)^{T}}(\mu A) \mathbf{u}^{(1)} \leq \boldsymbol{y}^{T} M^{-1} \boldsymbol{y}$. 

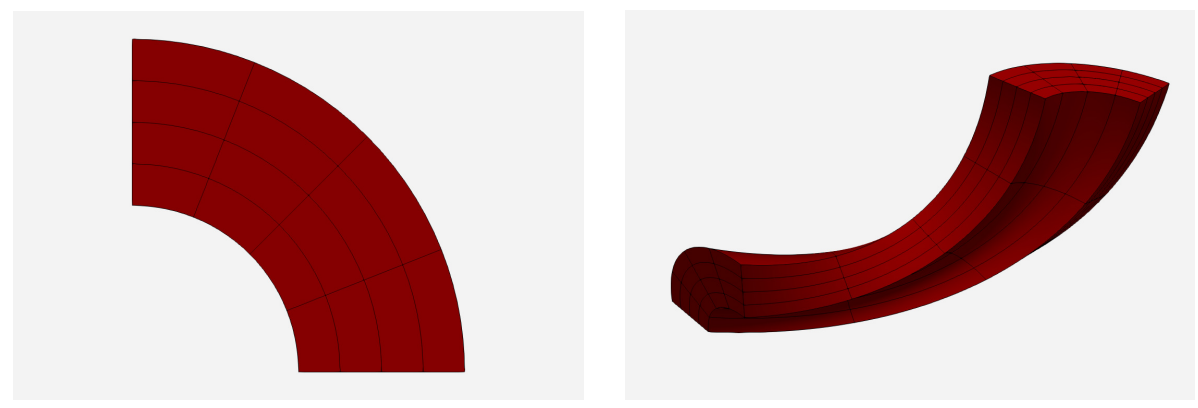

FIG. 7.1. Quarter annulus (left) and twisted pipe (right) geometries.

A bound for $\boldsymbol{u}=\boldsymbol{u}^{(1)}+\boldsymbol{u}^{(2)}$ and $p=p^{(2)}$ then yields $\boldsymbol{u}^{T}(\mu A) \boldsymbol{u}+p^{T}\left(\frac{1}{\lambda} C\right) p \leq$ $\left(10+\frac{12}{\beta^{2}}\right) \boldsymbol{y}^{T} M^{-1} \boldsymbol{y} \cdot \mathrm{\square}$

7. Numerical results. We now report the results of numerical experiments for the almost incompressible elasticity system (2.7) in two and three dimensions, discretized with isogeometric NURBS spaces with a uniform mesh size $h$, polynomial degree $p$, and regularity $k$. We use a zero Dirichlet boundary condition on the face $x=0$ of the reference cube, an inhomogeneous Neumann condition of the opposite face $x=1$, and zero Neumann conditions on all the other faces. The domain $\Omega$ is decomposed into $N$ non-overlapping subdomains of characteristic size $H$.

The discrete positive definite reformulation (4.2) is solved by the preconditioned conjugate gradient (PCG) method with the isogeometric block FETI-DP/BDDC preconditioner (5.1), with a zero initial guess and a stopping criterion of a $10^{-8}$ reduction of the Euclidean norm of the PCG residual. The first block $M_{p_{\Gamma}}^{-1}$ of our preconditioner (5.1) consists in the application of BDDC with deluxe scaling for the interface pressure $p_{\Gamma}$, scaled by the first Lamé parameter $\mu$. We have not found any good reason to use any primal variables for this sub-problem when using the BDDC deluxe variant and none have been used in any of the experiments reported in this section.

The second block $M_{\lambda_{\Delta}}^{-1}$ of (5.1) consists in the application of FETI-DP with deluxe scaling for the multiplier $\lambda_{\Delta}$. For the multiplier blocks, the primal constraints consists of all the dofs of the fat vertices, augmented, for 3D only, by the edge and face rigid body modes, orthogonalized with the SVD.

All parallel tests have been performed using PetIGA-MF $[12,40]$ as a discretization package; the solvers used are available in the latest release, 3.10, of the PETSc library [1], and have been contributed by Stefano Zampini (see also [47]). All the tests have been run on the parallel machine Shaheen-XC40 at KAUST.

In our tests, we study how the convergence rate of the BDDC preconditioner depends on $h, N, p$, and $k$. Results are reported on the total number of iterations (it) and the minimal and maximal eigenvalues $\left(\lambda_{m}\right.$ and $\left.\lambda_{M}\right)$ of the PCG operator.

7.1. A comparison with [36]. We first consider the AIE system on the unit square and compare the performance of our approach with that of [36]. We are close to the incompressible limit at $\nu=0.499$ and consider an increasing polynomial order $p$ with maximal continuity $k=p-2$, while keeping the mesh size $h=1 / 128$ and the subdomain diameter $H=1 / 8$ fixed. The Young's modulus is set to $E=1$.

Different choices for the block solvers are used as indicated in Table 7.1. Specifically, we work with a stiffness- or a deluxe-scaled FETI-DP multiplier preconditioner, as well as with three different variants for the pressure sub-system: a LU factorization 


\begin{tabular}{|c|c|c|c|c|c|c|c|c|c|}
\hline \multirow[b]{2}{*}{$p$} & \multicolumn{3}{|c|}{$p_{\Gamma}$ block: LU of $C_{\Gamma, \Gamma}$} & \multicolumn{3}{|c|}{$p_{\Gamma}:$ BDDC stiff. scaling } & \multicolumn{3}{|c|}{$p_{\Gamma}:$ BDDC deluxe } \\
\hline & $n_{i t}$ & $\lambda_{M}$ & $\lambda_{m}$ & $n_{i t}$ & $\lambda_{M}$ & $\lambda_{m}$ & $n_{i t}$ & $\lambda_{M}$ & $\lambda_{m}$ \\
\hline & \multicolumn{9}{|c|}{ Stiffness scaling FETI-DP for $\lambda_{\Delta}$ block } \\
\hline 2 & 23 & 2.2 & $2.5 \mathrm{e}-01$ & 22 & 2.2 & $2.8 \mathrm{e}-1$ & 22 & 2.2 & $3.73 \mathrm{e}-1$ \\
\hline 3 & 29 & 3.0 & $2.5 \mathrm{e}-01$ & 25 & 3.2 & $3.5 \mathrm{e}-1$ & 26 & 3.0 & $3.57 \mathrm{e}-1$ \\
\hline 4 & 49 & 6.7 & $1.5 \mathrm{e}-01$ & 37 & 6.8 & $3.2 \mathrm{e}-1$ & 39 & 6.6 & $3.64 \mathrm{e}-1$ \\
\hline 5 & 117 & 29.3 & $7.6 \mathrm{e}-02$ & 70 & 73.6 & $3.3 \mathrm{e}-1$ & 74 & 73.6 & $3.48 \mathrm{e}-1$ \\
\hline & \multicolumn{9}{|c|}{ Deluxe scaling FETI-DP for $\lambda_{\Delta}$ block } \\
\hline 2 & 25 & 2.3 & $2.4 \mathrm{e}-1$ & 23 & 2.3 & $2.8 \mathrm{e}-1$ & 23 & 2.3 & $3.67 \mathrm{e}-1$ \\
\hline 3 & 28 & 2.7 & $2.3 \mathrm{e}-1$ & 24 & 2.8 & $3.4 \mathrm{e}-1$ & 25 & 2.7 & $3.45 \mathrm{e}-1$ \\
\hline 4 & 33 & 2.7 & $1.5 \mathrm{e}-1$ & 33 & 4.8 & $3.2 \mathrm{e}-1$ & 26 & 2.7 & $3.48 \mathrm{e}-1$ \\
\hline 5 & 51 & 4.2 & $7.5 \mathrm{e}-2$ & 44 & 9.3 & $3.2 \mathrm{e}-1$ & 33 & 4.2 & $3.39 \mathrm{e}-1$ \\
\hline
\end{tabular}

Iteration counts and extreme eigenvalues for FETI-DP/BDDC block preconditioner for increasing $p=2, \ldots, 5$ and fixed $H=1 / 8, h=1 / 128, E=1, \nu=0.4999$, and using B-splines on the unit square. The preconditioner for the $\lambda_{\Delta}$ block is FETI-DP with stiffness scaling (top table) and FETI-DP deluxe (bottom table). The preconditioner for the $p_{\Gamma}$ block is $L U$ of the pressure mass sub-matrix $C_{\Gamma, \Gamma}$ (left column), BDDC with stiffness scaling (center column), and BDDC deluxe (right column).

\begin{tabular}{|c|rrr|rrr|}
\hline & \multicolumn{4}{|c|}{ FETI-DP for $\lambda_{\Delta}$ block alone } \\
& \multicolumn{3}{|c|}{ stiffness scaling } & \multicolumn{3}{c|}{ deluxe scaling } \\
$p$ & $n_{i t}$ & $\lambda_{M}$ & $\lambda_{m}$ & $n_{i t}$ & $\lambda_{M}$ & $\lambda_{m}$ \\
\hline 2 & 15 & 2.1 & 0.6 & 16 & 2.2 & 0.66 \\
3 & 15 & 3.0 & 0.9 & 16 & 2.7 & 0.71 \\
4 & 22 & 6.6 & 0.9 & 15 & 2.7 & 0.77 \\
5 & 44 & 29.3 & 0.9 & 19 & 4.1 & 0.68 \\
\hline
\end{tabular}

\begin{tabular}{|c|ccc|ccc|ccc|}
\hline & \multicolumn{10}{|c|}{ BDDC for $p_{\Gamma}$ block alone } \\
& \multicolumn{3}{|c|}{$\mathrm{LU}$} & & \multicolumn{3}{c|}{ stiffness scaling } & \multicolumn{3}{c|}{ deluxe scaling } \\
$p$ & $n_{i t}$ & $\lambda_{M}$ & $\lambda_{m}$ & $n_{i t}$ & $\lambda_{M}$ & $\lambda_{m}$ & $n_{i t}$ & $\lambda_{M}$ & $\lambda_{m}$ \\
\hline 2 & 11 & 0.80 & 0.37 & 11 & 0.9 & 0.43 & 11 & 0.9 & 0.43 \\
3 & 14 & 0.87 & 0.27 & 13 & 1.8 & 0.62 & 13 & 1.0 & 0.38 \\
4 & 19 & 0.96 & 0.15 & 27 & 4.4 & 0.36 & 15 & 1.2 & 0.35 \\
5 & 26 & 0.98 & 0.07 & 38 & 9.3 & 0.34 & 15 & 1.4 & 0.35 \\
\hline
\end{tabular}

Iteration counts and extreme eigenvalues for the separate sub-solvers of the FETI-DP/BDDC block preconditioner for increasing $p=2, \ldots, 5$. See Table 7.1 for details.

of the principal minor of the mass matrix associated with the interface variable $p_{\Gamma}$ and scaled by $\mu$, and stiffness- and deluxe-scaled BDDC solvers as outlined in Section 5.2. Detailed results on the convergence of the sub-solvers are reported in Table 7.2, which were obtained by applying the PCG method to the linear systems $G_{p_{\Gamma} p_{\Gamma}}$ and $G_{\lambda_{\Delta} \lambda_{\Delta}}$, using the preconditioners considered .

The results show that using an LU solver for the pressure block does not result in a minimal eigenvalue which is bounded from below and independent of $p$, while a BDDC pressure solver (using either stiffness or deluxe scaling) prove to be robust in this respect. In addition, a deluxe scaled FETI-DP method for the multiplier block seems necessary to mitigate the dependence of the condition number on the polynomial 


\begin{tabular}{|c|ccc|ccc|ccc|ccc|}
\hline & \multicolumn{3}{|c|}{$p=3, k=0$} & \multicolumn{3}{c|}{$p=3, k=1$} & \multicolumn{3}{c|}{$p=4, k=0$} & \multicolumn{3}{c|}{$p=4, k=2$} \\
$1 / h$ & $n_{i t}$ & $\lambda_{M}$ & $\lambda_{m}$ & $n_{i t}$ & $\lambda_{M}$ & $\lambda_{m}$ & $n_{i t}$ & $\lambda_{M}$ & $\lambda_{m}$ & $n_{i t}$ & $\lambda_{M}$ & $\lambda_{m}$ \\
\hline 32 & 20 & 1.6 & 0.28 & 21 & 2.0 & 0.31 & 23 & 2.2 & 0.27 & 24 & 2.8 & 0.28 \\
48 & 22 & 2.0 & 0.27 & 22 & 2.1 & 0.29 & 26 & 2.7 & 0.27 & 25 & 2.8 & 0.28 \\
64 & 24 & 2.3 & 0.27 & 23 & 2.3 & 0.29 & 28 & 3.1 & 0.27 & 25 & 2.8 & 0.27 \\
128 & 29 & 3.3 & 0.28 & 25 & 2.7 & 0.28 & 33 & 4.3 & 0.27 & 26 & 2.8 & 0.27 \\
256 & 34 & 4.6 & 0.28 & 29 & 3.5 & 0.28 & 37 & 5.8 & 0.27 & 29 & 3.5 & 0.27 \\
\hline \multicolumn{19}{c|}{ TABLE 7.3}
\end{tabular}

h-quasi-optimality of FETI-DP/BDDC block preconditioner with deluxe scaling for $2 D$ AIE system with $E=1 e+6, \nu=0.4999$, and with B-splines on the unit square. Iteration counts and extreme eigenvalues for increasing $1 / h=32, \ldots, 256$, different $p, k=0, \ldots, p-1$, and fixed $H=1 / 8(N=64)$.

\begin{tabular}{|c|ccc|ccc|ccc|}
\hline & \multicolumn{3}{|c|}{$p=2, k=0$} & \multicolumn{3}{c|}{$p=3, k=0$} & \multicolumn{3}{c|}{$p=3, k=1$} \\
$1 / h$ & $n_{i t}$ & $\lambda_{M}$ & $\lambda_{m}$ & $n_{i t}$ & $\lambda_{M}$ & $\lambda_{m}$ & $n_{i t}$ & $\lambda_{M}$ & $\lambda_{m}$ \\
\hline 16 & 25 & 2.1 & 0.23 & 31 & 3.1 & 0.22 & 29 & 2.7 & 0.24 \\
20 & 25 & 2.1 & 0.24 & 32 & 3.1 & 0.22 & 29 & 2.7 & 0.24 \\
24 & 26 & 2.4 & 0.24 & 33 & 3.3 & 0.23 & 29 & 2.7 & 0.23 \\
28 & 27 & 2.6 & 0.24 & 33 & 3.6 & 0.23 & 29 & 2.7 & 0.23 \\
32 & 28 & 2.8 & 0.24 & 34 & 3.9 & 0.23 & 30 & 2.7 & 0.23 \\
\hline
\end{tabular}

h-quasi-optimality of FETI-DP/BDDC block preconditioner with deluxe scaling for $3 D$ AIE system with $E=1 e+6, \nu=0.4999$, and with $B$-splines on the unit cube. Iteration counts and extreme eigenvalues for increasing $1 / h=16, \ldots, 32$, different $p, k=0, \ldots, p-1$, and fixed $H=1 / 4(N=64)$.

order. We note that the results for the stiffness scaled variant of the BDDC solver for the pressure sub-system were obtained by making all the fat vertices dofs primal. In fact, without a coarse space, the maximal eigenvalue of the pressure sub-system depended heavily on $p$, which in turn did not provide a satisfactory convergence rate for the global problem (data not shown).

7.2. Quasi-optimality test. We next consider the AIE system on the unit square and the unit cube, and study the performance of our block preconditioner when refining the mesh size $h$, while keeping the number of subdomains fixed at $N=64$. Different choices of the spline parameters $p$ and $k$ are tested as indicated in Tables 7.3 and 7.4. The Lamé parameters used in these tests are given by $E=1 e 6, \nu=0.4999$.

The results show that, for both two and three dimensions, our method behaves as predicted by the theory, since it presents a logarithmic growth in terms of both PCG iterations and condition number (given by the ratio $\lambda_{M} / \lambda_{m}$ ). For the cases $(p=4, k=2)$ in two dimensions, and $(p=3, k=1)$ in three dimensions, the method has proven optimal, since all these quantities remain almost constant when $h$ is refined. Such results can be attributed to our choice of using a very rich coarse space for the multiplier block; minimal coarse spaces, as presented in [37] for the compressible case, are outside the scope of the present work, and can be the subject of future research.

7.3. Polynomial order dependence test. We next report on the performance of our algorithm for different values for the spline parameters $p$ and $k$, while keeping the number of subdomains $(N=64)$ and the mesh related quantities $h$ and $H$ fixed: specifically $H=1 / 8, h=1 / 128$ in two dimensions and $H=1 / 4, h=1 / 20$ in three. The Lamé parameters used in the tests are given by $E=1 e 6, \nu=0.4999$. 


\begin{tabular}{|l|ccc|ccc|ccc|ccc|}
\hline & \multicolumn{3}{|c|}{$k=0$} & \multicolumn{3}{c|}{$k=1$} & \multicolumn{3}{c|}{$k=2$} & \multicolumn{3}{c|}{$k=3$} \\
$p$ & $n_{i t}$ & $\lambda_{M}$ & $\lambda_{m}$ & $n_{i t}$ & $\lambda_{M}$ & $\lambda_{m}$ & $n_{i t}$ & $\lambda_{M}$ & $\lambda_{m}$ & $n_{i t}$ & $\lambda_{M}$ & $\lambda_{m}$ \\
\hline 2 & 23 & 2.3 & 0.28 & \multicolumn{1}{c}{} & & & & & & & \\
3 & 29 & 3.3 & 0.28 & 25 & 2.7 & 0.28 & & & & & & \\
4 & 33 & 4.3 & 0.27 & 27 & 3.2 & 0.28 & 26 & 2.8 & 0.27 & & & \\
5 & 36 & 5.2 & 0.27 & 34 & 4.4 & 0.28 & 28 & 3.6 & 0.27 & 33 & 4.2 & 0.27 \\
\hline
\end{tabular}

p-dependence of FETI-DP/BDDC block preconditioner with deluxe scaling for $2 D$ AIE system with $E=1 e+6, \nu=0.4999$, and with $B$-splines on the unit square. Iteration counts and extreme eigenvalues for $p=2, \ldots, 5$, different continuity $k=0, \ldots, p-1$, and fixed $H=1 / 8(N=64), h=$ $1 / 128$.

Results for the parametric domains are given in Tables 7.5 and 7.6 for the twoand three-dimensional case, respectively. Tables 7.7 and 7.8 contains results obtained by working on the quarter annulus and the twisted pipe geometries shown in Figure 7.

The results show that the number of iterations and the condition numbers of the proposed method appear to depend logarithmically on the polynomial order $p$, even for the case involving geometrical mappings and for both two and three dimensions.

\begin{tabular}{|c|ccc|ccc|ccc|}
\hline & \multicolumn{3}{|c|}{$k=0$} & \multicolumn{3}{c|}{$k=1$} & \multicolumn{3}{c|}{$k=2$} \\
$p$ & $n_{i t}$ & $\lambda_{M}$ & $\lambda_{m}$ & $n_{i t}$ & $\lambda_{M}$ & $\lambda_{m}$ & $n_{i t}$ & $\lambda_{M}$ & $\lambda_{m}$ \\
\hline 2 & 25 & 2.2 & 0.24 & & & & & & \\
3 & 32 & 3.1 & 0.22 & 29 & 2.7 & 0.24 & & & \\
4 & 39 & 4.9 & 0.20 & 39 & 4.8 & 0.23 & 63 & 16.3 & 0.21 \\
\hline
\end{tabular}

p-dependence of FETI-DP/BDDC block preconditioner with deluxe scaling for $3 D$ AIE system with $E=1 e+6, \nu=0.4999$, and with $B$-splines on the unit cube. Iteration counts and extreme eigenvalues for $p=2,3,4$, different continuity $k=0, \ldots, p-1$, and fixed $H=1 / 4(N=64), h=$ $1 / 20$.

\begin{tabular}{|c|ccc|ccc|cccc|ccc|}
\hline & \multicolumn{3}{|c|}{$k=0$} & \multicolumn{3}{c|}{$k=1$} & \multicolumn{3}{c|}{$k=2$} & \multicolumn{3}{c|}{$k=3$} \\
$p$ & $n_{i t}$ & $\lambda_{M}$ & $\lambda_{m}$ & $n_{i t}$ & $\lambda_{M}$ & $\lambda_{m}$ & $n_{i t}$ & $\lambda_{M}$ & $\lambda_{m}$ & $n_{i t}$ & $\lambda_{M}$ & $\lambda_{m}$ \\
\hline 3 & 40 & 9.4 & 0.28 & 30 & 4.1 & 0.28 & & & & & & \\
4 & 44 & 11.1 & 0.28 & 34 & 5.8 & 0.28 & 31 & 4.0 & 0.27 & & & \\
5 & 48 & 12.9 & 0.28 & 40 & 7.6 & 0.28 & 35 & 5.1 & 0.27 & 35 & 5.2 & 0.27 \\
\hline
\end{tabular}

p-dependence of FETI-DP/BDDC block preconditioner with deluxe scaling for $2 D$ AIE system with $E=1 e+6, \nu=0.4999$, on the quarter annulus geometry as given in Fig. 7, left panel. Iteration counts and extreme eigenvalues for $p=3,4,5$, different degree of smoothness, $k=0, \ldots, p-1$, and fixed $H=1 / 8(N=64), h=1 / 128$.

The number of iterations and the maximal eigenvalue of the PCG method certainly increase for the mapped domains, especially for the quite distorted map of the cube given in the right panel of Figure 7. By looking at the convergence properties of the sub-solvers for the latter case (see Table 7.9), we see that the slower convergence depends on the multiplier block, while the pressure sub-solver has proven very efficient. Improvements, with up to 3 times fewer iterations, (data not shown) can be obtained by accelerating the multiplier sub-solver with a few Chebyshev iterations. Adaptive primal spaces for the displacements solver may also be considered in future work. 


\begin{tabular}{|c|ccc|rrr|rccc|}
\hline & \multicolumn{3}{|c|}{$k=0$} & \multicolumn{3}{c|}{$k=1$} & \multicolumn{3}{c|}{$k=2$} \\
$p$ & $n_{i t}$ & $\lambda_{M}$ & $\lambda_{m}$ & $n_{i t}$ & $\lambda_{M}$ & $\lambda_{m}$ & $n_{i t}$ & $\lambda_{M}$ & $\lambda_{m}$ \\
\hline 3 & 126 & 105.3 & 0.26 & 122 & 84.1 & 0.25 & & & \\
4 & 143 & 130.9 & 0.26 & 127 & 100.4 & 0.26 & 142 & 103.1 & 0.24 \\
\hline
\end{tabular}

p-dependence of FETI-DP/BDDC block preconditioner with deluxe scaling for $3 D$ AIE system with $E=1 e+6, \nu=0.4999$, on the twisted pipe geometry as given in Fig.7, right panel. Iteration counts and extreme eigenvalues for $p=3,4$, different degree of smoothness, $k=0, \ldots, p-1$, and fixed $H=1 / 4(N=64), h=1 / 20$.

\begin{tabular}{|r|rcc|ccc|ccc|}
\hline & \multicolumn{3}{|c|}{$k=0$} & \multicolumn{3}{c|}{$k=1$} & \multicolumn{3}{c|}{$k=2$} \\
$p$ & $n_{i t}$ & $\lambda_{M}$ & $\lambda_{m}$ & $n_{i t}$ & $\lambda_{M}$ & $\lambda_{m}$ & $n_{i t}$ & $\lambda_{M}$ & $\lambda_{m}$ \\
\hline & \multicolumn{8}{c|}{$\lambda_{\Delta}$ block } \\
3 & 93 & 105.2 & 0.54 & 74 & 83.9 & 0.66 & - & - & - \\
4 & 101 & 130.6 & 0.53 & 84 & 100.3 & 0.61 & 86 & 102.9 & 0.74 \\
\hline \multicolumn{8}{|c}{$p_{\Gamma}$ block } \\
3 & 20 & 2.6 & 0.33 & 17 & 1.7 & 0.35 & - & - & - \\
4 & 27 & 4.6 & 0.33 & 21 & 2.6 & 0.35 & 20 & 2.4 & 0.35 \\
\hline
\end{tabular}

Sub-solvers convergence study for the results provided in Table 7.8. Twisted pipe geometry as given in Fig. 7, right panel.

7.4. Weak scalability test. We close this Section by reporting, in Table 7.10, results of a weak scalability test in the parametric domain, obtained by considering a fixed number of elements in each direction and each subdomain (32x32 elements in two dimensions, $7 \times 7 \times 7$ for three dimensional tests), and by uniformly increasing the number of subdomains in each direction. As a result, the mesh parameters $H$ and $h$ decrease at the same rate, and their ratio is kept constant while the number of subdomains is increased. The Lamé parameters used in the tests are given by $E=$ $1 e 6, \nu=0.4999$; the spline tuples considered are $(p=4, k=2)$ in two dimensions and $(p=3, k=1)$ in three dimensions. The experimental results confirm the algorithmic scalability of our method, since the number of iterations remains constant as the number of subdomains, as well as the number of unknowns, is increased.

8. Conclusions. We developed and analyzed a block FETI-DP type domain decomposition algorithm for the solution of three-dimensional almost-incompressible elasticity discretized by isogeometric analysis techniques with a continuous pressure space. This work extends to the case of isogeometric analysis and almost incompressible elasticity the pioneering paper by $\mathrm{Tu}$ and $\mathrm{Li}$ [44], which focused on standard finite element discretizations of the Stokes system. We have explored different preconditioners for the pressure block, developing in particular an effective BDDC deluxe preconditioner without a coarse component. Theoretical results have shown that the resulting block preconditioner is quasi-optimal, with a polylogarithmic bound with respect to the ratio $H / h$, and algorithmically scalable with respect to the number of subdomains. Extensive parallel numerical results have validated the theoretical estimates and shown the robustness of the proposed method with respect to the polynomial order and regularity of the spline space. Future work may consider the construction and analysis of adaptive techniques for the choice of the primal constraints, in order to reduce the computational costs required by the solution of the 


\begin{tabular}{|c|ccc|}
\hline subd & $n_{i t}$ & $\lambda_{M}$ & $\lambda_{m}$ \\
\hline 10x10 & 29 & 3.6 & 0.26 \\
20x20 & 29 & 3.6 & 0.26 \\
30x30 & 29 & 3.6 & 0.26 \\
40x40 & 29 & 3.6 & 0.26 \\
50x50 & 28 & 3.6 & 0.26 \\
\hline
\end{tabular}

\begin{tabular}{|c|ccc|}
\hline subd & $n_{i t}$ & $\lambda_{M}$ & $\lambda_{m}$ \\
\hline $4 \times 4 \times 4$ & 29 & 2.7 & 0.23 \\
$5 \times 5 \times 5$ & 29 & 2.7 & 0.23 \\
$6 \times 6 \times 6$ & 29 & 2.7 & 0.23 \\
$7 \times 7 \times 7$ & 29 & 2.7 & 0.23 \\
$8 \times 8 \times 8$ & 29 & 2.7 & 0.23 \\
$9 \times 9 \times 9$ & 29 & 2.7 & 0.23 \\
$10 \times 10 \times 10$ & 29 & 2.7 & 0.23 \\
\hline
\end{tabular}

TABLE 7.10

Weak scalability of FETI-DP/BDDC block preconditioner with deluxe scaling for the AIE system with $E=1 e+6, \nu=0.4999$. Iteration counts and extreme eigenvalues for increasing number of subdomains (subd). Left: $2 D$ case with $p=4, k=2, H / h=32$. Right: $3 D$ case with $p=3, k=1$, $H / h=7$.

coarse problem.

Acknowledgements. For computer time, this research used also the resources of the Supercomputing Laboratory at King Abdullah University of Science \& Technology (KAUST) in Thuwal, Saudi Arabia.

\section{REFERENCES}

[1] S. Balay, S. Abhyankar, M.F. Adams, J. Brown, P. Brune, K. Buschelman, L. Dalcin, A. Dener, V. Eijkhout, W.D. Gropp, D. Kaushik, M.G. Knepley, D.A. May, L. Curfman McInnes, R. T. Mills, T. Munson, K. Rupp, P. Sanan, B.F. Smith, S. Zampini, and H. Zhang. PETSc Web page, http://www.mcs.anl.gov/petsc, 2018.

[2] Y. Bazilevs, L. Beirão da Veiga, J.A. Cottrell, T.J.R. Hughes, G. Sangalli. Isogeometric analysis: approximation, stability and error estimates for $h$-refined meshes. Math. Mod. Meth. Appl. Sci., 16, 1-60, 2006.

[3] L. Beirão da Veiga, D. Cho, L.F. Pavarino and S. Scacchi. Overlapping Schwarz methods for isogeometric analysis. SIAM J. Numer. Anal., 50: 1394-1416, 2012.

[4] L. Beirão da Veiga, D. Cho, L. F. Pavarino, S. Scacchi. BDDC preconditioners for Isogeometric Analysis. Math. Mod. Meth. Appl. Sci. 23(6): 1099-1142, 2013.

[5] L. Beirão da Veiga, D. Cho, L. F. Pavarino and S. Scacchi. Isogeometric Schwarz preconditioners for linear elasticity systems. Comput. Methods Appl. Mech. Engrg. 253: 439-454, 2013.

[6] L. Beirão da Veiga, L.F. Pavarino, S. Scacchi, O.B. Widlund, and S. Zampini. Isogeometric BDDC preconditioners with deluxe scaling. SIAM J. Sci. Comp. 36 (3): A1118-A1139, 2014.

[7] L. Beirão da Veiga, L.F. Pavarino, S. Scacchi, O.B. Widlund, and S. Zampini. Adaptive Selection of Primal Constraints for Isogeometric BDDC deluxe Preconditioners. SIAM J. Sci. Comp. 39 (1): A281-A302, 2017.

[8] H. Benhassine and A. Bendali. A non-overlapping domain decomposition method for continuous-pressure mixed finite element approximations of the Stokes problem. ESAIM Math. Model. Numer. Anal., 45: 675-696, 2011.

[9] D. Boffi, F. Brezzi and M. Fortin, Mixed Finite Element Methods and Applications. Computational Mathematics, vol. 44, Springer-Verlag, Berlin, 2013.

[10] A. Bressan and G. Sangalli. Isogeometric discretizations of the Stokes problem: stability analysis by the macroelement technique. IMA J. Numer. Anal., 33 (2), 629-651, 2013.

[11] J.A. Cottrell, T.J.R. Hughes, and Y. Bazilevs. Isogeometric Analysis. Towards integration of $C A D$ and FEA. Wiley, 2009.

[12] L. Dalcin, N. Collier, P. Vignal, A.M.A. Cortes, and V.M. Calo. PetIGA: A framework for high-performance isogeometric analysis. Comp. Meth. Appl. Mech. Engrg., 308: 151-181, 
2016.

[13] C.R. Dohrmann. A Preconditioner for substructuring based on constrained energy minimization. SIAM J. Sci. Comput., 25: 246-258, 2003.

[14] C.R. Dohrmann and O.B. Widlund. Some recent tools and a BDDC Algorithm for 3D problems in H(curl). Proceedings of the 20th Int. Conf. on Domain Decomposition Methods. Held in San Diego, CA, February 7-11, 2011. Springer LNCSE, 91: 15-26, 2013.

[15] C.R. Dohrmann and O.B. Widlund. A BDDC Algorithm with deluxe scaling for threedimensional H(curl) problems. Comm. Pure Appl. Math., 69 (4): 745-770, 2016.

[16] M. Donatelli, C. Garoni, C. Manni, S. Serra-Capizzano and H. Speleers. Symbol-based multigrid methods for Galerkin B-spline isogeometric analysis. SIAM J. Numer. Anal., 55 (1): 31-62, 2017.

[17] M. Dryja, M.V. Sarkis and O.B. Widlund. Multilevel Schwarz methods for elliptic problems with discontinuous coefficients in three dimensions. Numer. Math., 72: 313-348, 1996.

[18] K. Gahalaut, J. Kraus, and S. Tomar. Multigrid Methods for Isogeometric Discretization. Comp. Meth. Appl. Mech. Engrg., 253: 413-425, 2013.

[19] P. Gervasio, L. Dedé, O. Channon, A. Quarteroni. Comparing isogeometric analysis and spectral element methods: accuracy and spectral properties. MOX report 21/2018, Politecnico di Milano, 2018.

[20] P. Goldfeld, L.F. Pavarino, and O.B. Widlund. Balancing Neumann-Neumann preconditioners for mixed approximations of heterogeneous problems in linear elasticity. Numer. Math., 95 (2): 283-324, 2003.

[21] C. Hofer. Analysis of discontinuous Galerkin dual-primal isogeometric tearing and interconnecting methods. Math. Models Methods Appl. Sci. 28 (1): 131-158, 2018.

[22] C. Hofer. Parallelization of continuous and discontinuous Galerkin dual-primal isogeometric tearing and interconnecting methods. Comput. Math. Appl. 74 (7): 1607-1625, 2017.

[23] C. Hofer and U. Langer. Dual-primal isogeometric tearing and interconnecting solvers for multipatch dG-IgA equations. Comput. Methods Appl. Mech. Engrg. 316: 2-21, 2017.

[24] C. Hofreither and S. Takacs. Robust multigrid for isogeometric analysis based on stable splittings of spline spaces. SIAM J. Numer. Anal. 55 (4): 2004-2024, 2017.

[25] C. Hofreither. A black-box low-rank approximation algorithm for fast matrix assembly in isogeometric analysis. Comput. Methods Appl. Mech. Engrg. 333: 311-330, 2018.

[26] H. H. Kim and C.-O. Lee. A two-level nonoverlapping Schwarz algorithm for the Stokes problem: Numerical study. Comput. Methods Appl. Mech. Engrg., 223/224: 153-160, 2012.

[27] A. Klawonn and L. F. Pavarino. Overlapping Schwarz methods for mixed linear elasticity and Stokes problems. Comput. Methods Appl. Mech. Engrg., 165: 233-245 (1998).

[28] A. Klawonn and O.B. Widlund. FETI and Neumann-Neumann iterative substructuring methods: connections and new results. Comm. Pure. Appl. Math., 54: 57-90, 2001.

[29] A. Klawonn and O.B. Widlund. Dual-primal FETI methods for linear elasticity. Comm. Pure Appl. Math., 59: 1523-1572, 2006.

[30] S.K. Kleiss, C. Pechstein, B. Jüttler, and S. Tomar. IETI - Isogeometric Tearing and Interconnecting. Comp. Meth. Appl. Mech. Engrg., 247-248: 201-215, 2012.

[31] J. Li and X. Tu. A nonoverlapping domain decomposition method for incompressible Stokes equations with continuous pressures. SIAM J. Numer. Anal. 51 (2): 1235-1253, 2013.

[32] J. Li and X. Tu. A unified dual-primal finite element tearing and interconnecting approach for incompressible Stokes equations. Internat. J. Numer. Methods Engrg. 94 (2): 128-149, 2013.

[33] J. Li and O. B. Widlund. FETI-DP, BDDC, and block Cholesky methods. Int. J. Numer. Meth. Engrg., 66 (2): 250-271, 2006.

[34] J. Mandel and C.R. Dohrmann. Convergence of a balancing domain decomposition by constraints and energy minimization. Numer. Lin. Alg. Appl., 10 (7): 639-659, 2003.

[35] D.-S. Oh, O.B. Widlund, S. Zampini, and C.R. Dohrmann. BDDC algorithms with deluxe scaling and adaptive selection of primal constraints for Raviart-Thomas vector fields. Math. Comp. 87: 659-692, 2018.

[36] L.F. Pavarino and S. Scacchi. Isogeometric block FETI-DP preconditioners for the Stokes and mixed linear elasticity systems. Comput. Methods Appl. Mech. Engrg., 310: 694-710, 2016.

[37] L.F. Pavarino, S. Scacchi, O.B. Widlund and S. Zampini. Isogeometric BDDC Deluxe preconditioners for linear elasticity. Math. Mod. Meth. Appl. Sci. 28(7): 28(7): 1337-1370, (2018).

[38] C. Pechstein and C. R. Dohrmann, A unified framework for adaptive BDDC. Electr. Trans. Numer. Anal, 46: 273-336, 2017.

[39] G. Sangalli and M. Tani. Isogeometric preconditioners based on fast solvers for the Sylvester 
equation. SIAM J. Sci. Comput., 38 (6), A3644-A3671, 2016.

[40] A. F. Sarmiento, A. M. Cortes, D. A. Garcia, L. Dalcin, N. Collier, V. M. Calo. PetIGA-MF: a multi-field high-performance toolbox for structure-preserving B-splines spaces. J. Comput. Sci., 18: 117-131, 2017.

[41] J. Sistek, B. Sousedik, P. Burda, J. Mandel, and J. Novotny. Application of the parallel BDDC preconditioner to the Stokes flow. Comput. \&S Fluids, 46: 429-435, 2011.

[42] S. Takacs. A robust multigrid method for the time-dependent Stokes problem. SIAM J. Numer. Anal. 53 (6): 2634-2654, 2015.

[43] A. Toselli and O.B. Widlund. Domain Decomposition Methods: Algorithms and Theory. Computational Mathematics, Vol. 34. Springer-Verlag, Berlin, 2004.

[44] X. Tu and J. Li. A FETI-DP Type Domain Decomposition Algorithm for Three-Dimensional Incompressible Stokes Equations. SIAM J. Numer. Anal., 53(2): 720-742, 2015.

[45] O.B. Widlund and C.R. Dohrmann. BDDC deluxe Domain Decompositions. Proceedings of 22nd Int. Conf. on Domain Decomposition Methods. Held in Lugano, Switzerland, September 16-20, 2013. Springer LNCSE, 104: 93-103.

[46] O.B. Widlund, L.F. Pavarino, S. Scacchi, and S. Zampini. Preconditioners for Isogeometric Analysis and Almost Incompressible Elasticity. Submitted to the proceedings of the twenty-fifth international conference of domain decomposition held in St. John's, Newfoundland, Canada, July 23 to 27, 2018.

[47] S. Zampini. PCBDDC: a class of robust dual-primal preconditioners in PETSc. SIAM J. Sci. Comput., 38 (5), S282-S306, 2016.

[48] S. Zampini. Adaptive BDDC Deluxe Methods for H(curl). Proceedings of the 23rd Int. Conf. on Domain Decomposition Methods. Held on Jeju Island, Korea, July 6-10, 2015. Springer LNCSE, 116: 285-293, 2017.

[49] S. Zampini and X. Tu. Multilevel balancing domain decomposition by constraints deluxe algorithms with adaptive coarse spaces for flow in porous media. SIAM J. Sci. Comput., 39: A1389-A1415, 2017.

[50] S. Zampini, P. S. Vassilevki, V. Dobrev and T. Kolev . Balancing Domain Decomposition by Constraints Algorithms for Curl-conforming Spaces of Arbitrary Order. Proceedings of the 24th Int. Conf. on Domain Decomposition Methods. Held on Svalbard, Norway, February 6-10, 2017. Springer LNCSE, 125: 103-116, 2018. 